\title{
Coccidioidomycosis: A Contemporary Review
}

Nancy F. Crum

Received: January 25, 2022 / Accepted: February 4, 2022 / Published online: March 1, 2022

(C) The Author(s) 2022

\begin{abstract}
Coccidioidomycosis, colloquially known as Valley Fever, is an invasive dimorphic fungal infection caused by Coccidioides immitis and $C$. posadasii. The fungi are found in the arid desert soils of the southwestern US, as well as in parts of Mexico and Central and South America. Acquisition is typically via inhalation of arthroconidia which become airborne after both natural (e.g., earthquakes, dust storms, and fires) and human-related events (e.g., military maneuvers, recreational activities, agriculture, and construction). The incidence of infection in increasing likely a result of both climatic and populational changes. Further, the recognized geographic distribution of Coccidioides spp. is expanding, as cases are being diagnosed in new areas (e.g., eastern Washington, Oregon, and Utah). Most coccidioidal infections are asymptomatic (60\%); however, approximately one-third develop a pulmonary illness which is a leading cause of communityacquired pneumonia in highly endemic areas. Uncommonly (0.5-2\% of cases), the infection disseminates to extrapulmonary locations (e.g., skin, bones/joints, and the central nervous system), and is most commonly seen among
\end{abstract}

\section{N. F. Crum ( $\square)$}

Infectious Diseases Department, Scripps Health

System, San Diego, CA, USA

e-mail: nancy32red@yahoo.com persons with cellular immunodeficiencies (e.g., transplant recipients, HIV, and pregnancy) and non-Caucasian races (especially African Americans and Filipinos). The diagnosis of coccidioidomycosis requires astute clinical suspicion and laboratory findings, including positive serology, cultures, and/or histopathology results. Treatment is warranted among persons with pneumonia who have risk factors for complicated disease and among those with extrapulmonary disease. Novel antifungals with improved fungicidal activity and rapidity of action with fewer side effects and drug interactions are needed. Preventive strategies (e.g., education regarding the disease, dust avoidance, mask wearing, including among select groups, antifungal prophylaxis, and surveillance laboratory testing) are advised for residents and travelers to endemic areas. Currently, no preventive vaccine is available. Coccidioidomycosis has been recognized for over a century, and an expanding wealth of knowledge has been gained regarding this emerging infectious disease which will be reviewed here.

Keywords: Coccidioidomycosis; Coccidioides; Valley fever; Endemic mycosis; Review 


\section{Key Summary Points}

Coccidioidomycosis is a regional disease of global importance, given the potential exposures of an increasing numbers of travelers and residents into endemic areas.

The geographic distribution of Coccidioides spp. and the number of cases has been recently increasing.

While most cases are asymptomatic, coccidioidomycosis is a common cause of community acquired pneumonia in highly endemic areas and may lead to disseminated, life-threatening disease especially among immunosuppressed persons as well as among those of African American or Filipino ethnicities.

Novel antifungals with increased potency against Coccidioides spp. as well as randomized comparative clinical trials to determine the preferred agent(s) are needed.

The search for a vaccine for coccidioidomycosis continues however an approved vaccine for human use is likely decades away.

\section{INTRODUCTION}

Just over 120 years ago, a new pathogen called Coccidioides was described-first thought to be a parasite and later re-classified as a dimorphic fungus. Intense study of the novel disease commenced in the southwestern US, focused in areas of highest endemicity including the San Joaquín Valley of California (hence the name, "Valley Fever") and southern Arizona. Both the disease incidence and distribution of Coccidioides spp. have expanded, making it an emerging infectious disease of notable importance. Although coccidioidomycosis cases are acquired in specific and limited geographic areas, the disease and its management have global implications given its increasing incidence and potential exposures to those who venture into endemic areas for recreational or occupational activities. This paper describes the current understanding of the history, ecology, epidemiology, risk factors, clinical manifestations, diagnosis, and management of this important reemerging disease. This article is based on previously conducted studies and does not contain any new studies with human participants or animals performed by the author.

\section{HISTORY}

The first recognition of the disease occurred in 1892 by Alejandro Posadas, an intern in Buenos Aires, Argentina [1]. He described a soldier with a debilitating skin condition including a fungallike mass and other ulcerative skin lesions of the face. A biopsy showed organisms resembling the protozoan, Coccidia, and hence the newly described disease was originally thought to be of parasitic origin. A similar clinical case occurred in the late 1890s in California of a manual laborer with similar facial skin lesions, who succumbed to his illness and on autopsy was found to have diffuse involvement of the lungs, lymph nodes, liver, peritoneum, and other body locations. Biopsies showed protozoa-like structures as well as a mold; the latter was unfortunately discarded as a contaminant [2]. Casper Gilcrest and Emmet Rixford subsequently named the organism Coccididioides (resembling Coccidia) and immitis (not mild) [3]. Soon thereafter, William Ophuls and Herbet Moffitt demonstrated Koch's postulates by inoculating the organism from human samples into animals with the subsequent development of the disease. Further, they determined that the organism was not a protozoan but rather a dimorphic fungus [4]. Interestingly, two other regional fungal infections in the US, histoplasmosis and blastomycosis, were also originally misidentified as protozoal infections before they too were classified as dimorphic fungi.

The infection caused by $C$. immitis was thought to be both rare and nearly always fatal for the first three decades after its discovery. However, in 1929, medical student, Harold 
Chope, at what would later become Stanford University was accidentally exposed in the laboratory. Although the student developed a respiratory illness including right upper lobe pneumonia and erythema nodosum (EN) (and his condition was feared to be fatal based on the original descriptions of patients succumbing to disseminated forms of the disease), he recovered. As such, it was recognized that the condition was not always severe or fatal (hence the species name, immitis, was incorrect) prompting additional studies of the pathogenicity of infection. In fact, during the following years, it was recognized that natives of the San Joaquin Valley frequently suffered from a similar illness of fevers, pneumonia, and EN ("Valley Fever"), and often fully recovered. A Coccididioides skin test was subsequently developed and showed a high prevalence of asymptomatic residents in the Valley were test-positive, supporting the view that most infected persons were not severely affected by the organism. These investigations revealed that most infections were mild and self-resolving. Although the name, Coccidioides immitis, remains, the meanings of the genus and species are incorrect descriptors for both the pathogen and the disease [2].

Subsequent epidemiologic evaluations by Smith et al. in the 1930s and 1940s focused on new arrivals into the central valleys of California (e.g., military personnel, immigrants from the Midwest during the "Dust Bowl", and Japanese prisoners of war). These investigations provided landmark data on the disease's incidence, risk factors (including non-Caucasian ethnicity), transmission route, incubation period, and clinical manifestations $[5,6]$.

Over time, endemic areas outside California were discovered, and, in fact, the highest rates of disease were subsequently documented in south/central Arizona. New areas of endemicity continue to be described in other parts of the USA, and also exist in other countries (Mexico, and Central and South America). The history of coccidioidomycosis continued to evolve, with two distinct species described when, in 2002, $C$. immitis was divided to include a second species, C. posadasii, named after the original describer of the disease [7].

\section{MYCOLOGY}

Coccidioides spp. are fungi within the Ascomycete division, Eurotiomycetes class, and Onygenales order [8]. By genetic analyses, the genus is most closely related to ascomycetes, including Blastomyces dermatitidis, Histoplasma capsulatum, and Paracoccidioides spp. It is estimated that the genus Coccidioides has existed for 40-50 million years, and that the species split into the current two species approximately 5 million years ago [9].

The two species, C. immitis and C. posadasii, are morphologically identical and share 90\% homology regarding their predicted proteins [8]. To date, the two species are thought to be similar phenotypically in terms of clinical manifestations, in vitro susceptibilities, and response to antifungal therapies. Whether genotypic variation between the fungal species could contribute to virulence differences is in its infancy of investigation, and future research is awaited. The two species have different, but overlapping, geographic regions: C. immitis exists in California, Utah, and Washington State, whereas C. posadasii is found in Nevada, Arizona, New Mexico, West Texas, Mexico, and Central and South America. However, both species exist in southern California and Baja California. Although New Mexico is a state in which $C$. posadasii primarily occurs, a recent analysis found both species present [10], suggesting that clear-cut geographic boundaries often do not exist and may be changing over time. Additionally, there are described hybrid strains, suggesting that the two species exchange genetic material [11].

Coccidioides spp. are classified as dimorphic fungi that exist in a mycelium or spherule form. Both forms of the organism undergo asexual growth, but there is likely a sexual phase of the organism's life cycle that has not been specifically observed. In the environment (i.e., soil) and on agar media (when infected human material is cultured in the laboratory), the organism grows as mycelia by apical extension. Alternating hyphal cells undergo autolysis, resulting in individual, frail barrel-shaped 3- to $5-\mu \mathrm{m}$ cells called arthroconidia. During the 
rainy season, the mycelia grow rapidly in the soil and can remain viable for many years [12]. When soil is disturbed by natural or human activities, the spores (i.e., the arthroconidia) can become airborne, leading to disease after inhalation.

Once inside the body (human or mammalian), the arthroconidia transform into spherical cells consisting of an outer wall and are called spherules. Although the spherule is the predominant form of the dimorphic fungus, hyphal forms with infectious arthroconidia have been noted in lung cavities and draining sinuses. Within each spherule, multiplication via synchronous division of the nuclei and cytoplasm occurs, and 2- to 4- $\mu \mathrm{m}$ daughter cells, called endospores, are formed. As the spherule matures, the outer wall thins and ruptures, sending 100-300 endospores into the body. Each endospore can then form another spherule within $\sim 4$ days, leading to rapid increases in the fungal burden as it repeats its life cycle [13].

Infection in both humans and animals primarily occurs within the lung. Spread outside of the parenchyma (i.e., disseminated disease) can occur via hematogenous (most commonly) or lymphatic spread.

\section{EPIDEMIOLOGY}

\section{Areas of Endemicity and Its Changing Distribution}

Coccidioides is found in the dry, arid regions of the Western Hemisphere [14], concentrated within the north and south $40^{\circ}$ latitudes. The organism has not been isolated from soils outside of the Western hemisphere, hence the disease is restricted to those who reside or travel to endemic areas with rare exception. Specifically, the organism seems to prefer the arid, alkaline desert soil of the Lower Sonoran Life Zone with an estimated annual rainfall of 5-20 inches (c). $12-50 \mathrm{~cm}$ ) during the winter season followed by hot, dry summers. During Smith's epidemiologic studies, he eloquently described the increase in infection rates with heavy rainfalls during the winter/spring months followed by hot, dry summers [5].
The specific locations that characterize these climatic and soil features occur in several states located in the southwestern US, including Arizona, California, New Mexico, Utah, Nevada, and west Texas. Of note, $\sim 60 \%$ or more of all coccidioidomycosis cases in the U.S. occur in Arizona and $\sim 30 \%$ in California. In Arizona, infections primarily occur in three counties within the southern part of the state-Maricopa, Pinal, and Pina. Together, these three counties account for $\geq 80 \%$ of cases in the state. In California, the most endemic areas include the seven counites of the San Joaquin Valley, with the highest endemicity described in Kern County [9]. Compared to Arizona, the disease distribution in California is more diffuse and involves major metropolitan areas, including San Diego and Los Angeles. Regarding seasonality, most cases in California and Arizona occur in the late summer and fall.

Endemic areas of Coccidioides spp. in the US appear to be expanding. Studies have recently demonstrated that, in Nevada, cases are not only found in the southern part but also in the north, including Four Corners. Non-contiguous areas of endemicity have been described, including Dinosaur National Monument in Utah $[15,16]$ and in eastern Washington State [17]. Other unidentified pockets of endemicity may exist, given the evolving geographic distribution of Coccidioides spp. For instance, Coccidioides spp. DNA was identified in soil samples from central Oregon [18], with at least one case acquired in the state [19]. Evidence of shifting areas of endemicity over time is also supported by fossil data; for example, a bison estimated to have lived $\sim 8500$ years ago was discovered in Nebraska containing Coccidioides spherules in its bony remains [20]. These data suggest that the geographic range of Coccidioides has changed over time and likely will continue to do so. High winds can carry coccidioidal spores for long distances (75 miles or more) [21]; further, it is possible that animals can carry the organism into new areas and succumb, introducing the fungus into the soils there. As such, practitioners should be cognizant of the possibility of coccidioidomycosis occurring outside the currently identified endemic zones. 
In addition to the US, Coccidioides exists in parts of other countries, including Mexico near the US border in the areas of Sonora, Nayarit, Jalisco, and Michoacán, as well as in central areas, including Coahuila, Durango, and San Luis Potosi), Central America (Guatemala, Honduras, and Nicaragua), and South America (Argentina, Bolivia, Paraguay, Venezuela, Columbia, and Brazil).

The current understanding of the distribution of Coccidioides spp. has primarily relied on clinical case surveillance. The specific requirements of the soil for the growth and maintenance of Coccidioides spp. remain incompletely defined, and the identification of the fungus in soil samples from "endemic areas" is often spotty and inconsistent. Even if infections are acquired from a specific location, the soil samples may not yield the organism [22]. Of note, the recovery of the organism from soil is challenging, as culture techniques (using semiselective agar) and mouse inoculation (e.g., peritoneal injection of soil extracts) have notable limitations. PCR technology using novel testing (e.g., CocciENV) may improve the sensitivity of detection [23].

While studies on the soil characteristics that support the existence of Coccidioides spp. are ongoing, another question is the potential role of desert animals, including rodents, in its life cycle and growth. Some studies have shown that the fungus can use the soil as the sole source of nutrients [12], while others have demonstrated a higher recovery of Coccidioides spp. from soil acquired from rodent burrows $[24,25]$. Desert animals may become infected (as shown by coccidioidal granulomas in their lungs), and their subsequent demise may result in the introduction of spherules back to the soil with transformation to the mycelial form and subsequent growth within the soil. While many types of animals can develop coccidioidal infection (including sea life, such as dolphins, dogs, cats, livestock, zoo animals, alpacas, and primates, to name a few), desert rodents that burrow and make their home within the soil are most likely to contribute to the fungus' life cycle. In sum, data suggest that Coccidioides spp. can persist alone in the soil for years, while desert animals may amplify its growth and carry the organism into new territories.

\section{Incidence Trends Over Time}

In addition to expanding areas of Coccidioides endemicity, the number of cases has increased. The Centers of Disease Control and Prevention analyzed data from the National Notifiable Diseases Surveillance System and found that coccidioidomycosis increased substantially from 5.3 per 100,000 population in endemic areas within five states (Arizona, California, Nevada, New Mexico, and Utah) in 1998 to 42.6 per 100,000 in 2011 [26]. Other studies have also demonstrated rising rates, including a study in California showing an increase from 2.4 to 18.8 cases per 100,000 persons from 2000 to 2018 , an $800 \%$ increase [27-30]. While the number of coccidioidomycosis cases annually in the US has been estimated as 150,000 , this is likely an underestimate due to rising incidence rates and missed diagnoses (discussed below); in fact, recent data suggest that the annual number is likely $\sim 350,000$ [31]. Data regarding incidence trends and case numbers from other countries are not readily available.

Regarding incidence rates, large-scale investigations in the southwestern U.S. were undertaken in the 1940s and 1950s. For example, Smith demonstrated that $\sim 25-50 \%$ of military personnel/recruits training in the San Joaquin Valley seroconverted (via skin testing) in a single year [5]. A study published in the 1940s found that skin test reactivity increased with length of residency in endemic areas, and found that $\sim 80 \%$ of children tested positive after $\geq 10$ years $[2,32]$. Studies in the 1950 s of long-term residents in the Arizona counties of Pima (Tucson), Maricopa (Phoenix), and Pinal showed a skin test positivity prevalence of $50 \%$. Similarly, in very high-risk areas of California, including Kern (Bakersfield), Tulare, and Kings counties, the positive rate was $50-70 \%[33,34]$. While these data suggest a high penetrance of the infection among natives and persons training in highly endemic areas, these data are more than half a century old and newer data on incidence and prevalence trends are needed [9]. 
Unfortunately, a complete understanding of the changing incidence over the past century is lacking, given sparse case detection and reporting over much of the history of disease. However, to assist with a more accurate determination of the burden of disease, in 1995, coccidioidomycosis became reportable in the US, and, in 2014, laboratory-based reporting was added in the state of California.

Recent annual incidence rates are estimated at $1-3 \%$ in the endemic areas of southern Arizona and central California [21, 35, 36]. Studies in military trainees performing high-risk maneuvers noted an incidence of $6-32 \%$ annually, although case numbers were small, limiting the precision of estimates [37]. Finally, an outbreak investigation among military personnel training in an endemic area over a 6 -week period noted high rates of infection (10 of $22,45 \%$ ) thought to due to high-inoculum exposures [22]. Further data from prospective surveillance studies in endemic areas are needed. While existing data suggest that the contemporary incidence of coccidioidomycosis in the US is not greater than in the 1940s/1950s, the rate has varied over time, and has clearly been increasing during the last decade.

\section{Reasons for the Increasing Numbers of Cases}

The reasons for the recent increasing case numbers are likely several-fold, including both host and environmental factors. Host factors include modifiable risks related to recreational/ occupational activities and travel into highly endemic areas, as well as non-modifiable risks, including aging and complex underlying health conditions summarized below. Environmental factors include climate changes and natural events. Finally, improved case detection may be contributing to the increasing numbers of reported cases.

Human activities that can cause the fungus to become airborne [30] increase the risk for infection. Occupations such as agricultural work; archeology as demonstrated by the outbreak at Dinosaur National Park [15, 16, 38]; firefighting, especially when using hand tools and working in dusty conditions; and construction work, including on solar power farms [39-42], have all been associated with infection acquisition [43]. Military personnel stationed in endemic areas are also at particular risk, as demonstrated by the initial studies of Smith in the 1940s [5], with more recent literature substantiating the ongoing risk for military personnel participating in outdoor military training [22, 44, 45]. Overall, occupational exposures constitute a significant risk for coccidioidomycosis, as exemplified by a review of 47 coccidioidomycosis outbreaks during 1940-2015, of which 25 (53\%) were related to occupational exposures [39]. Of occupationalrelated outbreaks, $50 \%$ were related to construction [39].

Recreational activities in desert environments of endemic regions also place people at increased risk. Activities associated with infections have included model airplane flying, outdoor track and field events, filming movies, and all-terrain vehicle or go-cart riding/racing [46-48]. An increasing number of residents and visitors participating in these activities likely contributes to the rising incidence of the disease.

Host factors for infection that are non-modifiable include older ages, and the rising number of elderly persons with multiple comorbidities in endemic areas. Further, visitors or new residents naive to the disease are at-risk groups. Persons with deficient cellular immunity due to underlying conditions (e.g., AIDS and posttransplant recipients) or receipt of immunosuppressant therapies (e.g., steroid use of $\geq 20 \mathrm{mg}$ daily for $\geq 2$ weeks, chemotherapy, TNF-alpha blockers, or other immune suppressants) are at particular risk, and are increasing in number within the general population. Finally, the obesity epidemic has increased the number of persons with comorbidities including diabetes which are risk factors for symptomatic disease.

Environmental factors are also important drivers for rising coccidioidal rates. Urban development of land (that was previously sparsely inhabited) into major metropolitan areas, such as Phoenix, Arizona, has contributed to incident infections. While urbanization formed 
paved streets and buildings that covered the previously open desert soil (with less potential exposure to airborne arthroconidia), the massive influx of people into these areas and nearby recreational activities on open soil may have tipped the balance towards increasing numbers of infections. Further, several prisons have been built in endemic areas (e.g., the central valleys of California) with notable outbreaks among the incarcerated population, groups in which minority ethnicities are overrepresented and who simultaneously have greater risk for symptomatic and severe disease [39].

Climatic factors and natural events are also likely a factor. The incidence of infection increases during the dry summer months after previous periods of heavy winter/spring rains. As such, climate changes (with very dry summers) may alter the geographic distribution and case counts. Specifically, it has been hypothesized that global warming may cause areas of endemicity to expand northward, enlarging the geographic range of the fungus [49]. Natural events, such as earthquakes [50], dust storms $[51,52]$, and fires $[43,53]$, have been linked to infections [54]. Some experts predict that, due to climatic and other environmental changes, the incidence of coccidioidomycosis may increase by $164 \%$ by 2050 [49].

Finally, Coccidioides disease recognition and diagnostic testing can affect incidence rates. Currently, there are no definitive data supporting this as a major driver since coccidioidomycosis remains an underdiagnosed condition $[10,55,56]$. Changes in surveillance definitions and reporting may also be contributory.

\section{PATHOGENESIS AND RISK FACTORS FOR DISEASE}

\section{Transmission Routes}

The vast majority of coccidioidal infections are acquired via inhalation of airborne arthroconidia. Rarely primary cutaneous inoculation with the development of skin infection [57], as well as acquisition via organ transplantation (due to infected donor lungs, liver, and kidneys) leading to disseminated disease, have been described [58-60]. Rare cases of neonatal transmission have been reported, and are thought to be mainly due to aspiration of infectious vaginal secretions during birth [61]. Person-to-person transmission is otherwise not a feature of the disease.

\section{Pathogenesis After Exposure}

After inhalation, arthroconidia are deposited in the terminal bronchiole and are subsequently transformed into spherules. A single inhaled arthroconidia can result in infection. Within a spherule, hundreds of endospores are formed that are subsequently released, thereby exponentially multiplying the burden of disease. A T-cell immune response to fungus can contain the infection within the lungs, resulting in an asymptomatic or minimally symptomatic respiratory illness [62]. Sequelae infection includes a pulmonary nodule or cavity, although most infections fully resolve.

Disease severity and dissemination are dependent on the vigor of the innate and adaptive immune responses. Initial immune responses to the fungus involve neutrophils, macrophages, and dendritic cells. Cellular immunity [Th (T-helper) cells and Th17] is critical for infection control within the lungs and prevention of dissemination [62], as demonstrated by the marked risk for extrapulmonary spread among persons with T-cell deficiencies. Antibodies are not thought to be protective. Persons who have low titers of complement fixation (CF) antibodies, and a robust delayed T-cell response due to active Th1, Th17, and interferon gamma production typically do well and are at low risk for dissemination. As such, a positive and strong coccidioidal skin test reaction early during the disease course portends a good prognosis.

\section{Risk Factors for Coccidioidomycosis}

Early during the AIDS epidemic, HIV-infected persons with low CD4 counts $\left(<250\right.$ cells $\left./ \mathrm{mm}^{3}\right)$ were at heightened risk for coccidioidal infection, which highlighted the role of T-lymphocytes in fungal containment [63, 64]. More 
recent data have similarly demonstrated that lower CD4, higher HIV RNA levels, and AIDS diagnosis are associated with more severe disease [65]. As expected, the incidence and severity of Coccidioides among HIV-infected persons has declined with the availability of antiretroviral therapy [66, 67].

Other groups at risk for coccidioidal infection due to cellular immunodeficiency include transplant recipients and those receiving immunosuppressant agents, such as high doses of corticosteroids, anti-tumor necrosis factor medications, chemotherapy, or other immunomodulating therapies utilized for rheumatologic conditions or inflammatory bowel disease. Women in their 3rd trimester of pregnancy or early post-partum period are also at increased risk, but whether this is due to pregnancy-related cellular immune suppression and/or hormonal changes is unclear [68, 69]. Infection among these groups can be a primary infection or reactivation; the latter is most likely among transplant recipients who present with infection during the first-year posttransplantation.

As noted above, host resistance to coccidioidomycosis requires a robust cell-mediated immunity, including adequate production of Th1 cytokines (interleukin-12, and IFN-gamma) as well as the appropriate regulation and functionality of Th1/Th2 responses and IL-12/IFNgamma cytokine axes [70]. Therefore, persons with genetic mutations (congenital or acquired) in these immune components, including interferon- $\gamma$ deficiencies, a gain of function mutation in STAT1, and interleukin 12 deficiency [71-74], are at risk for complicated disease. Humoral immunity plays a lesser role, evidenced by the fact that persons with immunoglobulin deficiencies do not appear to be at particular risk.

Diabetes mellitus has been found to be a risk factor for severe and complicated pulmonary infection (e.g., cavitary), and those with uncontrolled blood sugars may also be at risk for dissemination [75]. In addition to the medical conditions outlined above, studies have noted that males and older adults have an increased risk of disseminated disease [30]. Of note, extrapulmonary disease is more common in adults than children.

Additionally, there is a long-recognized risk of disseminated disease among certain racial groups. Smith, during his landmark studies in the 1940s, recognized that African Americans were particularly affected, and subsequent studies have confirmed this observation. Filipinos also have a higher risk $[5,30,32,76]$. There are some data to suggest an increased risk of severe/disseminated disease among Hispanics [76, 77], with less data supporting an association among Asians and Native Americans $[10,78]$. Concurrent comorbidities and socioeconomic factors (access to care and occupation) may explain some of the racial differences, but not all suggesting an underlying genetic predisposition [30]. The exact genetic factor(s) has not been defined, but is being investigated in an ongoing study, "Pathogenesis and Genetics of Disseminated or Refractory Coccidioidomycosis" conducted at the University of Arizona through an National Institutes of Health (NIH) Grant (NCT02190266).

\section{CLINICAL MANIFESTATIONS}

\section{Pulmonary Infection}

An estimated $60 \%$ of infections are asymptomatic and do not lead to clinical presentation, whereas the remainder develop a respiratory illness following exposure. The incubation period from exposure to onset of respiratory symptoms is typically $1-3$ weeks. Of the projected 150,000 infections annually (based on older data), an estimated 50,000 are symptomatic and require medical evaluation, 10,000-20,000 are diagnosed as coccidioidomycosis, 2000-3000 have pulmonary sequelae, 600-1000 have extrapulmonary disease, and 160 result in death $[79,80]$.

In highly endemic areas, coccidioidomycosis is a leading cause of community-acquired pneumonia (CAP). For example, studies conducted in Arizona have found that Coccidioides spp. are responsible for $17-29 \%$ of all CAP cases $[81,82]$. Those with coccidioidomycosis typically have a clinical presentation that is 
indistinguishable from community-acquired bacterial pneumonia (CABP). Symptoms of coccidioidal pneumonia include cough, chest pain, dyspnea, and fever. Weight loss, night sweats, and headaches are often present. Fatigue may be especially prominent, and may continue for several months after the acute illness and occasionally can be debilitating.

A study comparing those with acute pulmonary coccidioidomycosis to CABP found similar clinical presentations, although fever was more common and cough less common in CABP compared to coccidioidomycosis. Those with coccidioidomycosis were more likely to have radiographic upper lobe disease, the presence of a pulmonary nodule, hilar or mediastinal adenopathy, and/or erythema nodosum, although each were overall uncommon and present in a minority of coccidioidal infections $[80,83]$. Coccidioidomycosis may lead to peripheral eosinophilia, but this too is uncommon. Finally, those with coccidioidomycosis may have a more protracted clinical course compared with CABP.

A common triad of symptoms, often referred to as "desert rheumatism", includes fever, EN, and arthralgias, along with the typical respiratory symptoms listed above. The arthralgias are typically symmetrical and involve the lower extremities, but do not lead to significant joint effusions. Both the skin and joint findings are due to immune-mediated responses rather than disseminated infection. The triad occurs most commonly in women and is usually associated with a good prognosis [5].

Infrequently, pulmonary infection may manifest as diffuse pulmonary infiltrates or miliary disease, most commonly among immunosuppressed persons. Respiratory failure and septic shock may occur. Sequalae of pulmonary infections include nodules, cavities, and chronic fibrocavity pneumonia which develop in a minority of cases, typically $5 \%$ of those with a pulmonary presentation. Cavities close to the pleura may rupture, causing a hydropneumothorax with or without an associated bronchopleural fistula leading to an empyema. Persons with uncontrolled diabetes have an increased risk for severe lung disease and/or cavity formation.

\section{Extrapulmonary Disease}

Disseminated disease is defined as involvement outside the pulmonary system and pleura space. The most common sites of disseminated disease include the skin, bones, joints, and central nervous system (CNS). While $0.5-2 \%$ of infected persons develop disseminated disease based on the literature, the exact percentage depends on the underlying host characteristics of the population. For example, dissemination can occur in $30-50 \%$ of heavily immunosuppressed patients [80]. The time frame from initial infection to dissemination is variable but is typically counted in weeks to months. Disseminated coccidioidomycosis can be a mimicker of many other conditions and can be a diagnostic challenge [76]. Patients with disseminated disease may have no respiratory symptoms and a normal chest radiograph (CXR).

The most benign form of disseminated disease is cutaneous involvement. There is no specific lesion type associated with the disease, but may include verrucose lesions, ulcers, and abscesses. There is a predilection for the nasolabial fold, but lesions can occur at any site. Skin lesions are often chronic and non-healing despite antibiotic courses and debridement procedures. Unlike disseminated skin involvement which contains fungal elements, other cutaneous findings that occur during the initial, acute coccidioidal infection, such as a macular diffuse rash, EN, or erythema multiforme are due to immunologic phenomena and are not due to the direct invasion of the fungus itself.

Joint involvement (most commonly knees followed by ankles and wrists) typically presents with effusion and synovitis. Dissemination of the fungus into the joints differs from desert rheumatism, which is immune-mediated and is typically associated with arthralgia alone without evidence of an active infectious process. Bony involvement commonly involves the axial skeleton. Vertebral disease, especially of the lumbar area, is a common location, and may be complicated with associated paravertebral, epidural, or psoas abscesses. Disease of the appendicular skeleton can also occur with or without associated joint involvement. 
CNS coccidioidomycosis is the most serious form of disseminated disease and can manifest as headaches, confusion, gait disturbance, change in mental status, or focal neurologic deficits. Basilar involvement can occur (like tuberculosis), and may result in cranial nerve abnormalities. Complications include hydrocephalus and vasculitic events, such as stroke.

While skin, joint, bone, and CNS involvement are the most common locations of extrapulmonary disease, any site can be infected with Coccidioides spp. Unusual locations described in case reports have included endocarditis [84], prostate abscess [85], peritonitis [86], intraocular [87], and thyroid infection [88], to name just a few.

After an initial coccidioidal infection, there appears to be lifelong immunity and an inability to acquire a new infection. Of note, this fungal infection can lie 'dormant' in the body and reactivate years later, causing disseminated disease among persons who subsequently become immunosuppressed (e.g., transplant recipients receiving ongoing immunosuppressants).

\section{DIAGNOSIS}

\section{Overview}

The diagnosis of coccidioidomycosis requires a high index of suspicion and consideration for this infection among those residing in or travelling from an endemic area. Pulmonary coccidioidomycosis often goes unrecognized, as the initial presenting symptoms are often assumed to be due to a bacterial or viral cause. Even providers in endemic areas may not specifically test for Coccidioides due to unfamiliarity or the perceived unimportance of the disease. Establishing the diagnosis of pulmonary coccidioidomycosis is important to avoid unnecessary antibiotic courses, provide the patient and their family with a specific diagnosis, enable education regarding the disease, and ensure appropriate follow-up for potential complicated or disseminated disease. The challenges in making the diagnosis of disseminated disease are notable, given the wide variety of signs and symptoms as noted above. Further, the diagnosis may be especially elusive among patients presenting outside of endemic areas, signifying the importance of a careful travel history for establishing the presence of a fungal infection in these cases.

Even among providers in endemic areas, the diagnosis of coccidioidomycosis may not be considered or may be missed. A survey conducted in New Mexico noted that $70 \%$ or more of physicians were uncertain about the diagnosis or treatment of coccidioidomycosis [10]. Further, a nationwide survey that queried providers if they frequently tested patients presenting with CAP for coccidioidomycosis found that only $3.7 \%$ responded affirmatively. Among providers in Arizona and California, 32\% and $7 \%$, respectively, stated that they frequently tested for the pathogen [55]. Similar low percentages were noted among a study in southern California [56].

Providers worldwide should be familiar with this mycosis, as cases have been noted among returning travelers to Canada [89], Asia [90, 91], Europe [92, 93], India [94], and other locations [95]. Further, infections have occurred after only minimal exposure to an endemic area, including cases occurring after a layover in the Phoenix airport or a single drive across the central valley of California.

A study showed that the median time from seeking healthcare to the diagnosis of coccidioidomycosis was 38 days (range 1-1654 days), and that $70 \%$ had another condition diagnosed before coccidioidal testing occurred (of whom $83 \%$ were prescribed antibacterial medications) [19]. This paper also highlighted that these time frames were extended among visitors who then traveled back to a non-endemic area prior to disease onset [19].

\section{Approach to the Diagnosis}

The diagnosis of coccidioidomycosis relies on a combination of epidemiologic information, clinical findings, physical examination, and laboratory/radiology data. All patients should have a careful history and physical 
examination, including evaluation for possible disseminated disease.

A CAR is routinely performed. CAR findings of coccidioidal pulmonary infection include most commonly a unilateral infiltrate, hilar adenopathy, and/or a small pleural effusion. Mediastinal adenopathy may be noted and can signify an increased for disseminated disease [76]. Pleural effusions when they occur are typically exudative, and may contain eosinophils, but are typically culture-negative unless a pleural biopsy is obtained (similar to tuberculosis). Polymerase chain reaction (PCR) of the pleural fluid has poor sensitivity and does not perform better than culture alone [96]. Adenosine deaminase levels are usually not elevated levels [96].

Any areas of focal complaints or examination abnormalities should be further evaluated usually with computed tomography (CT) or magnetic resonance imaging (MRI). An MRI is preferred for bony or spinal involvement. If there is concern for diffuse bony involvement, a radionuclide bone scan is useful. Those with joint effusions should undergo arthrocentesis and surgical evaluation for operative management. Similarly, those with spinal disease should have MRI imaging and neurosurgical evaluation. Finally, suspicious skin lesions should be biopsied for culture and histopathologic examinations.

Patients with significant headaches or neurologic findings concerning for brain/meningeal involvement should undergo brain MRI imaging, and, if deemed safe, a lumbar puncture (LP) performed. Brain imaging cannot be utilized to exclude coccidioidal meningitis (CM), hence an LP is needed for evaluation. Of note, an LP is specifically recommended among patients with unusual, persistent, or worsening headaches, those with altered mental status, new focal neurologic deficit, or unexplained nausea/vomiting [80]. Of note, headache is common during the initial presentation and often resolves within a week or so; in this setting, an LP is not specifically recommended unless the headache remains persistent or there are additional indications for LP. The LP should include measurement of the opening pressure and cerebrospinal fluid (CSF) glucose, protein, cell counts, and differential, fungal culture, and coccidioidal complement fixation (CF) titer. Coccidioides antigen testing can be considered. Findings consistent with CM include low-grade pleocytosis with lymphocytic predominance, elevated protein, and variable (low-normal) glucose findings. Occasionally, eosinophilia is noted and can suggest the diagnosis but is overall uncommon. CSF cultures are infrequently $(\sim 25 \%)$ positive, and the diagnosis of CM typically relies on abnormal CSF parameters as well as positive $\mathrm{CF}$ and/or antigen test results.

\section{Basic Laboratory Tests}

Patients with all forms of coccidioidomycosis often have elevated C-reactive protein and erythrocyte sedimentation rate levels, as well as hyperglobulinemia. The white blood count is variable, and the differential may occasionally reveal eosinophilia, but is often absent. The procalcitonin level is typically normal in cases of coccidioidal pneumonia [97]. Hypercalcemia has been described occasionally in disseminated cases due to expression of a parathyroid hormone-related peptide related to granulomatous inflammation [98].

Serum $(1 \rightarrow 3)-\beta$-d-glucan $(B G)$ is useful for diagnosing fungal infections such as Aspergillus and Pneumocystis. A study of the utility of BG (using a cutoff value of $\geq 80 \mathrm{pg} / \mathrm{ml}$ ) among a diverse group of hospitalized coccidioidomycosis patients found a sensitivity of $44 \%$ (and a specificity of $91 \%$ ), a positive predictive value of $82 \%$, and negative predictive value of $64 \%$. However, in acute pulmonary coccidioidomycosis, testing showed only a $19 \%$ positivity rate. BG levels were more likely elevated among disseminated cases but correlated poorly with serum coccidioidal CF titers [99].

\section{Specific Coccidioidal Tests}

The presumptive diagnosis of coccidioidomycosis is most frequently made with the detection of anticoccidioidal antibodies in the serum or cerebrospinal fluid. Serologic testing remains the most utilized method for diagnosing the disease. Enzyme immunoassay (EIA) tests are 
widely available and provide timely results, hence are the most frequently used test for initial screening. While detection of IgM and IgG antibodies by EIA are more sensitive than other available tests for detecting early disease [immunodiffusion tube precipitin test (IDTP), complement fixation titers], they are less specific and are hampered by the possibility of false-positive IgM results [100]. EIA-positive results should therefore be confirmed using another test, such as an IDTP, immunodiffusion complement fixation test, or complement fixing test, although these latter tests are often negative early in the disease; hence, follow-up testing (every $\sim 1-2$ weeks) is warranted in suspected cases [101]. Laboratories with expertise in these tests include the University of California, Davis (https://health.ucdavis.edu/ medmicro/cocci.html) [102].

Since it may take several weeks for antibodies to develop, and a negative serology (even using an EIA) does not rule out infection early in the disease course [101], repeat testing is recommended if the disease is suspected. The EIA IgM is initially positive, followed by detection of IgG antibodies. Most patients with disseminated disease will have detectable serum anti-coccidioidal IgG antibodies by EIA and/or immunodiffusion testing; however occasionally immunosuppressed persons will not mount detectable antibody responses [80] emphasizing the need for a high index of suspicion in this group and acquisition of clinical samples for culture/histopathologic examination.

Complement fixation (CF) titers which measures IgG antibody should be ordered in all cases of coccidioidomycosis and are important in assessing the burden of fungal infection and monitoring treatment responses (a decreasing titer indicates clinical improvement). CF testing involves placing a patient's serum with coccidioidal antigen in which immune complexes are formed (usually involving IgG immunoglobulin) that depletes complement. When antibodycoated red blood cells are added, the reduction in complement results in reduced lyses. The IgG antibodies are reported as titers (e.g., 1:2 is low and $1: 512$ is high). A titer $>1: 16$ has been associated with an increased likelihood for disseminated disease, with a caveat that CNS disease may have low titers, hence suspicion for CNS involvement should rely on symptoms, imaging, and CSF examination findings, rather than on CF titers alone [76]. Since CF titers are associated with disease progression and regression, they are very useful in monitoring the disease over time. CF titers are typically monitored every 6-8 weeks, especially early during the treatment course, but may be done less frequently over time.

A rapid lateral flow assay (LFA) for detection of coccidioidal antibodies with results in $1 \mathrm{~h}$ has been developed (Sōna; IMMY, Norman, OK, USA). Testing can be performed with minimal training and laboratory equipment at the point of care. A recent study of patients with early coccidioidomycosis showed a notably lower sensitivity for the LFA as compared to the available EIA tests. Specifically, compared to the standard blood EIA, the LFA demonstrated only a $31 \%$ sensitivity [103].

Coccidioidal antigen testing may be useful, since it does not require time (e.g., weeks) for the development of an antibody response and hence can be positive earlier (compared with antibody tests), and may be particularly useful among immunosuppressed persons who may not generate adequate antibody responses. Antigen testing may also be useful for the evaluation of CM [104]. Coccidioidal antigen testing is commercially available for use with blood, urine, or CSF samples.

Finally, PCR testing has been developed, and may also be positive early in disease before antibody production. Use of the PCR using respiratory and pleural samples have been explored in small studies [96, 105, 106]; however, this test is currently confined to the research setting, given the lack of robust clinical data supporting its use.

The definitive diagnosis of coccidioidomycosis, especially for disseminated disease or among immunosuppressed patients, often relies on culture and histopathology data. Cultures can be obtained from any source of suspected involvement, including sputum, bronchoscopy fluid, pleural fluid, synovial fluid, CSF, blood, or biopsies of the skin, bone, or other tissues. CSF and pleural cultures have low sensitivity $[96,104]$. Similarly, cultures of the blood are 
rarely positive, but case reports have described Coccidioides being isolated from the blood [107]. Fungal cultures of infected tissues (skin, bone, or joint fluid) have higher yield. Fungal culture growth occurs on most mycologic or bacteriology media at 5-7 days of aerobic incubation, demonstrating a white, non-pigmented mold. Since the mycelial form grows on agar media, inhalation of an open plate can be highly contagious. Thus, if Coccidioides is suspected, laboratory personnel should be informed, and the plates taped to avoid accidental exposure. If working with the organism in the laboratory, a biocontainment cabinet (biosafety level 3) should be utilized. A chemiluminescent DNA probe (Accuprobe; Gen Probe) is available to assist with the identification of the mold [108]. Differentiation between the two species is not commercially available, but can be conducted in research settings. Histopathology of tissue specimens using a variety of stains [most commonly the Grocott methenamine silver (GMS)] typically reveals granulomatous inflammation. Spherules are pathognomonic for coccidioidomycosis.

Coccidioidal skin testing was utilized early in the history of the disease for clinical and epidemiologic purposes. A positive test (induration of $>5 \mathrm{~mm}$ at $48 \mathrm{~h}$ ) indicates a past or current Coccidioides infection. In addition to helping with the diagnosis of an acute pulmonary infection with test positivity seen earlier than that of serologic testing, it may identify previously infected persons. Skin testing can also be a prognostic indicator, as anergy in the setting of a known coccidioidal infection predicts a higher risk of severe disease. While a skin test for coccidioidomycosis was unavailable for many years, a new reformulated spherule-derived test is currently available and FDA-approved in the US for adults 18-64 years old (Spherusol; https://nielsenbio.com/spherusol-hcp) [109].

\section{TREATMENT}

\section{Overview}

Antifungal treatment is determined by the type and extent of the coccidioidal infection (e.g., pulmonary vs. extrapulmonary; severe vs mild) and immunocompetence of the host. The Infectious Diseases Society of America (IDSA) has published specific guidelines on the management of coccidioidomycosis [80].

Available antifungal classes with activity against Coccidioides spp. include polyenes and azoles [110]. Currently, the only two FDA-approved medications for coccidioidomycosis are amphotericin B and ketoconazole; however, both are associated with the potential for significant adverse effects. Contemporary treatments include the off-label use of five currently available azoles: fluconazole, itraconazole, posaconazole, voriconazole, and isavuconazole.

\section{Polyenes}

Amphotericin B has been extensively utilized in the treatment of coccidioidomycosis over the last 50 years, and was initially the sole available agent. Amphotericin is currently available in multiple formulations, including amphotericin B deoxycholate, liposomal amphotericin B (L$\mathrm{AMB})$, amphotericin B colloidal dispersion, and amphotericin B lipid complex. All formulations are currently only available in intravenous (iv) forms. There are no human clinical trials comparing the various formulations; however, they are all considered efficacious based on animal data and clinical experience [111, 112]. Over time, the use of an L-AMB has become preferable given its better adverse event profile, especially among persons with underlying renal disease. All amphotericin formulations have potential side effects, including nephrotoxicity, hypokalemia, hypomagnesemia, phlebitis, fever, chills, body aches, and anemia. The typical dose of conventional amphotericin B for coccidioidomycosis is $0.7-1.0 \mathrm{mg} / \mathrm{kg}$ iv daily, and for lipid formulations is $3-5 \mathrm{mg} / \mathrm{kg}$ iv daily. Initially, therapy is typically given daily, and then transitioned to 3 times/week as the patient improves. Close monitoring of creatinine, magnesium, potassium, and hemoglobin levels is warranted. The duration of therapy is dependent on clinical improvement, but is typically for $\sim 3$ months, with subsequent transition to azole therapy. With the availability of the less toxic azole class, amphotericin is now 
reserved for severe, progressive coccidioidal or azole-unresponsive disease.

Utilization of amphotericin B via other routes than iv have been described. Since iv amphotericin B is ineffective for CM, it can be given by direct lumbar or cisternal injection or via lumbar, ventricular, or cisternal reservoirs; expert consultation is highly recommended given the complexities of this therapy [113]. Additionally, the use of amphotericin B-impregnated cement for bone or joint infections has been described in case reports, but its efficacy is unknown [114].

\begin{abstract}
Azoles
Ketoconazole was the first azole with demonstrable activity against Coccidioides spp., and is the only available azole approved by the FDA for treatment. However, this drug is no longer utilized in this role due to concerns for adverse events, including hepatotoxicity. The five azoles currently used to treat coccidioidomycosis are fluconazole, itraconazole, voriconazole, posaconazole, and isavuconazole. There are no randomized clinical trials that compare these azoles in terms of efficacy for coccidioidomycosis. The only comparative study performed evaluated fluconazole versus itraconazole, and was performed over 20 years ago [115]. This trial compared fluconazole $400 \mathrm{mg}$ daily and itraconazole $200 \mathrm{mg}$ twice daily, and demonstrated similar (within 20\%) outcomes. In a secondary analysis focused on skeletal disease, response rates were superior among those treated with itraconazole [115]. No other randomized studies have been conducted. Hence, decisions on which azole to prescribe are largely based on drug cost, adverse events/intolerances, drug absorption [therapeutic drug monitoring (TDM) is recommended for itraconazole, posaconazole, and voriconazole], and clinical experience. Given their lower costs and the robust clinical experience, fluconazole and itraconazole remain the preferred agents for treatment by the IDSA guidelines [80]; of note, in clinical practice, fluconazole is less toxic and easier to use than itraconazole.
\end{abstract}

All available azoles can cause hepatotoxicity and QTc prolongation (except isavuconazole which shortens the PR interval). A baseline electrocardiogram should be considered among those at risk for QT abnormalities, especially among those with cardiac disease, receipt of other QT-altering drugs, and patients with low potassium, magnesium, or calcium levels (electrolytes should be corrected before azole therapy is begun). Three azoles (itraconazole, voriconazole, and posaconazole) contain a cyclodextrin vehicle which may accumulate, especially in the setting of renal disease. Azoles have many potential drug interactions that require a careful review of concurrent medication history. Periodic monitoring of a complete blood count and comprehensive metabolic panel, which include liver tests, is recommended with prolonged use. Finally, azoles have been associated with teratogenicity, and hence should be avoided during the 1st trimester of pregnancy. For example, studies have shown that fluconazole may cause congenital craniosynostosis, skeletal deformities, heart defects, and spontaneous abortion [116, 117]. Hence, other than single-dose usage (which is not relevant for coccidioidal treatment), the FDA has categorized fluconazole as pregnancy class D. Similarly, other azoles should also be avoided in the 1st trimester $[66,80]$. Appropriate counseling and contraception should be provided to women of child-bearing age to avoid pregnancy while on azole therapy.

Fluconazole is typically considered the firstline agent, and can be utilized for all forms of coccidioidomycosis [80], given its excellent penetration into the brain, lungs, skin, and bone/joints. Typical dosing is $400 \mathrm{mg}$ po daily, but higher doses of $800-1200 \mathrm{mg}$ have been used for disseminated or severe disease. Advantages of fluconazole include its low cost, availability in both oral and intravenous formulations, no food requirement, long halflife, and excellent bioavailability. Fluconazole side effects (in addition to those listed above) include alopecia, xerosis, cheilitis, and arthropathy, which are often dose-related, with higher doses associated with more bothersome side effects. 
Itraconazole is the second most commonly prescribed azole to treat coccidioidomycosis, and is available as a capsule or oral solution. Dosing is typically $200 \mathrm{mg}$ po three times daily for 3 days, followed by $200 \mathrm{mg}$ po twice daily thereafter. Absorption is challenging, and can be improved by taking the capsule with a high fat meal and acidic beverage (Coke); the oral solution is taken on an empty stomach. The oral solution has greater bioavailability, but has more gastrointestinal side effects. TDM is recommended to ensure adequate drug levels; concentrations generally reach steady state by 2 weeks, and target concentrations are preferably $>2 \mathrm{mcg} / \mathrm{ml}$ with goal random level of 3-6 $\mathrm{mcg} / \mathrm{ml}$ [21]. Itraconazole has potential adverse side effects distinct from fluconazole, including hypertension, hypokalemia, sodium retention, and negative inotropic effects with resultant cardiac issues, and hence is best avoided in those at risk of heart failure [118]. Despite reported poor CSF and bone penetration, studies have shown itraconazole to be efficacious in the treatment of CNS and osseous forms of coccidioidomycosis.

In cases of intolerance to or failure of fluconazole and/or itraconazole, an alternate azole may be considered. Voriconazole is available in both intravenous and oral formulations, and is widely distributed throughout body including in the CSF. Dosing is $400 \mathrm{mg}$ twice daily $\times 1$ day and then $200 \mathrm{mg}$ twice daily thereafter, taken on an empty stomach. Some have alternatively recommended using weight-based dosing with a loading dose of $6 \mathrm{mg} / \mathrm{kg}$ every $12 \mathrm{~h} \times 1$ day, followed by $4 \mathrm{mg} / \mathrm{kg}$ every $12 \mathrm{~h}$ thereafter [21]. Voriconazole has distinct potential side effects, including visual disturbances and hallucinations, mental status alterations, alopecia, xerosis, periostitis, photodermatitis, and an increased risk for cutaneous malignancies including squamous cell carcinoma and melanoma [119]. Patients should be counseled to avoid the sun, utilize sunscreen and sun-protective clothing, and undergo skin cancer surveillance. Regarding adverse events, toxicity appears to be related to elevated serum drug levels influenced by the CYP-2C19 genotype, and hence TDM is recommended with a trough goal of $1-5 \mathrm{mcg} / \mathrm{ml}$. TDM has been shown to improve both the efficacy and safety of this antifungal agent [120]. Case reports and retrospective series have reported the use of voriconazole for both meningeal and nonmeningeal coccidioidal disease [121-124].

Posaconazole is available in both intravenous and oral forms. The oral form was initially available only as an oral solution; however, bioavailability was a problem. Currently, a delayed release (DR) oral tablet is available and preferable given its improved absorption. Typical dosing of the DR tablet is $300 \mathrm{mg}$ twice daily $\times 1$ day and then $300 \mathrm{mg}$ daily thereafter and is taken with food. Common side effects include gastrointestinal upset, hypokalemia, hypertension, peripheral edema, dry mouth, and headache. Additionally, posaconazole-related mineralocorticoid excess has been described, and presents with hypertension and hypokalemia attributed to the inhibition of $11 \mathrm{~b}$-hydroxylase or $11 \mathrm{~b}$-hydroxysteroid dehydrogenase 2 [125]. Hence, monitoring of blood pressure and potassium levels are recommended during therapy. Posaconazole levels should be monitored with a goal random level of 3-6 mcg/ml [21]. Posaconazole has been shown to penetrate most sites of the body, but exhibits poor CSF penetration [126]. Posaconazole has shown to be effective in the treatment of some refractory cases of coccidioidomycosis [121, 127-129].

Isavuconazole is the newest azole, and is available in both oral and IV formulations. Dosing is $372 \mathrm{mg}$ of isavuconazonium sulfate (the prodrug) every $8 \mathrm{~h}$ for $48 \mathrm{~h}$ followed by $372 \mathrm{mg}$ of isavuconazonium sulfate daily thereafter, with no food requirements (of note, $372 \mathrm{mg}$ of isavuconazonium sulfate is equivalent to $200 \mathrm{mg}$ of isavuconazole). Potential adverse events include gastrointestinal disorders (diarrhea and nausea/vomiting) and hypokalemia. This azole is unique in that it can lead to QTc shortening (vs. prolongation seen with other azoles). Given that the drug has high bioavailability, TDM is not required. A prospective study has demonstrated its efficacy in the treatment of primary Coccidioides infection and salvage therapy, including for refractory CM [130-133]. 
While azoles remain the most frequently utilized class for treatment of coccidioidomycosis, clinical failures can occur, and treatment response may be slow requiring months to even years of therapy. Whether Coccidioides isolates have become more resistant to azoles has been questioned. While antifungal susceptibility is not readily available in clinical laboratories, it can be performed in research settings. As such a study of 581 isolates from disparate geographic locations in North America were analyzed from 2001 to 2015. This study found elevated MIC values for fluconazole $(\geq 16 \mu \mathrm{g} / \mathrm{ml}, 37.3 \%$ of isolates; $\geq 32 \mu \mathrm{g} / \mathrm{ml}, 7.9 \%$ of isolates), itraconazole ( $\geq 2 \mu \mathrm{g} / \mathrm{ml}, 1.0 \%$ of isolates), posaconazole ( $\geq 1 \mu \mathrm{g} / \mathrm{ml}, 1.0 \%$ of isolates), and voriconazole ( $\geq 2 \mu \mathrm{g} / \mathrm{ml}, 1.2 \%$ of isolates). Voriconazole and posaconazole MICs were significantly lower than those of fluconazole, itraconazole, and amphotericin B $(p<0.0001)$. While the clinical relevance of these data is unknown as patient outcomes were not studied, the observed decreased in vitro susceptibility of fluconazole may explain the need for higher fluconazole doses and/or poor clinical responses to fluconazole in some cases [134]; further studies are needed.

\section{Other Antifungal Agents, New Drug Development, and Combination Therapy}

Other available antifungal agents include the echinocandins. This class has been evaluated as a potential treatment option since Coccidioides spp. contain $\beta-1,3$ glucan and this drug class inhibits beta-(1,3)-D-glucan synthase. There is variable in vitro susceptibility within the echinocandin class, with caspofungin having higher MICs compared to anidulafungin and micafungin [134]. Disappointingly, both in vitro and animal studies have demonstrated little activity against Coccidioides spp. [135-137]. Hence, echinocandins are not considered frontline agents for coccidioidomycosis, and should only be considered in the setting of combination salvage therapy.

There remains a need for newer fungicidal oral agents against Coccidioides spp. with improved potency and fewer side effects and drug interactions. Unfortunately, as a regional disease, coccidioidomycosis has not garnered significant attention to prompt randomized, clinical trials or specific drug development. Industry interest has been limited in recent decades, and the support of governmental or philanthropic organizations is needed. The development of new drugs for coccidioidomycosis was the focus of a recent FDA workshop [138].

While drug development for coccidioidomycosis has stalled, antifungal development in general has accelerated, and these drugs could be investigated regarding their potential role in the treatment of coccidioidomycosis. Recently, there are numerous agents in development, including improved formulations of older drugs as well as new drugs with novel mechanisms of action. For example, a new formulation of itraconazole (SUBA-itraconazole) has been developed and clinical studies are ongoing [139], as well as a novel oral amphotericin B formulation (iCo-019) [140]. Additionally, investigational antifungal agents are advancing in development, including Olorofim (formerly F901318), an orotomide (inhibitor of dihydroorotate dehydrogenase) with excellent in vitro activity against Coccidioides spp., in vivo efficacy demonstrated in a murine model for $\mathrm{CM}$, and human studies planned $[141,142]$. Nikkomycin $\mathrm{Z}$ is a chitin synthase inhibitor that has shown promising results in murine models of infection [143-145]. Additional agents include fosmanogepix (formerly APX001), new glucan synthase inhibitor (rezafungin), a fungal mitochondrial inhibitor (T2307), VT-1598, an investigational tetrazole that selectively inhibits fungal Cyp51A, and a histone deacetylase inhibitor (MGCD290) [138, 146]. Overall, we eagerly await novel antifungal therapies with anti-Coccidioides spp. activity, which ideally will provide more rapid anti-fungal effects, greater efficacy and cure rates, shorter treatment duration, and improved side effects and drug interaction profiles.

Combination antifungal therapy has been considered for a variety of fungal pathogens; however, clinical trials are lacking regarding its potential role in coccidioidomycosis. In the past, there has been a theoretical concern regarding combining an azole with amphotericin $\mathrm{B}$, since the former agent inhibits 
ergosterol in the cell membrane and thereby could interfere with the action of amphotericin binding and depleting ergosterol; however, this concern has not been confirmed in studies or clinical experience. Since it stands to reason that targeting multiple pathways using a combination of drugs may improve efficacy, animal studies have been conducted studying antifungals that act at different sites. For example, a murine model of coccidioidomycosis demonstrated that the combination of caspofungin and amphotericin B deoxycholate had synergistic effects with increased survival and decreased fungal burden compared to monotherapy with either treatment alone $[137,147]$. Whether this combination has a potential role in refractory coccidioidomycosis remains unclear, and further studies are needed. Investigational agents such as nikkomycin $\mathrm{Z}$ has demonstrated additive and synergistic effects in vitro when combined with azoles such as fluconazole [148]; clinical studies combining these agents are of interest once nikkomycin Z is available.

\section{Treatment Recommendations}

\section{Pneumonia}

Most immunocompetent patients with acute, uncomplicated pneumonia are not thought to require specific antifungal therapy and typically self-resolve their illness [80]. There are no placebo-controlled studies currently available to determine if antifungal treatment is of clinical benefit. Two retrospective studies did not demonstrate that early azole treatment compared with no treatment improved time to symptom resolution or risk for recurrence or dissemination [149, 150]. A phase IV randomized clinical trial was initiated to evaluate early fluconazole therapy versus placebo for CAP in endemic areas with a goal enrollment of 1000 subjects; unfortunately, the study was terminated early due to poor enrollment (only 72 participants enrolled during the first year) [151].

While some clinicians may advocate for treatment of all cases regardless of severity or host characteristics, data suggest caution to this approach. For example, in a trial that provide treatment with fluconazole or itraconazole, relapse nonetheless occurred in 18-28\% [115], questioning whether treatment positively affected outcomes. Further, some have questioned whether early treatment may adversely dampen immune responses, with data showing a lack of IgG response in some cases when azoles are administered early in infection; however, $\mathrm{T}$-cell responses and clinical outcomes were not studied [152].

For persons with coccidioidal pneumonia who have a debilitating infection or specific host factor(s), antifungal therapy is recommended [80] (Table 1). Symptoms to prompt antifungal therapy have included weight loss $>10 \%$ of body weight, intense night sweats $>3$ weeks, infiltrates of more than half of one lung or involving both lungs, prominent or persistent hilar or peritracheal adenopathy, CF titer $>1: 16$, symptoms lasting $>2$ months, need for hospitalization, or inability to work [80]. Additionally, patients with underlying host factor(s) that increases the risk for complicating disease, treatment is warranted in the setting of a symptomatic infection. These conditions include diabetes, a frail health status due to age or comorbidities, or an immunosuppressive condition (see risk factors above). Many experts also recommend treating those of non-Caucasian races, especially African Americans and Filipinos, due to their propensity to develop disseminated disease [80]. Patients with HIV infection who have a CD4 cell count of $<250$ cells $/ \mathrm{mm}^{3}$ should also be treated; of note, immune reconstitution inflammatory syndrome is thought to be uncommon and delaying antiretroviral therapy, while treating pulmonary coccidioidomycosis is not recommended [66]. Coccidioidomycosis among HIVinfected persons with CD4 cell counts of $\geq 250$ cells $/ \mathrm{mm}^{3}$ is treated similarly to those without HIV infection.

No clinical trials been performed to determine the optimal drug, dose, or duration for pulmonary coccidioidomycosis among persons warranting treatment. Antifungal treatment based on clinical experience and per the IDSA guidelines is typically with fluconazole $400 \mathrm{mg}$ daily (or itraconazole $200 \mathrm{mg}$ po twice daily) for 3-6 months [80]. The choice of fluconazole for 
Table 1 Treatment recommendations for coccidioidomycosis

\section{Clinical presentation}

Type of antifungal therapy

None

NA

Pneumonia, not debilitating and no risk factors for dissemination $^{\mathrm{b}}$

Pneumonia, debilitating or with risk factors for dissemination $^{\mathrm{b}}$

Diffuse pneumonia with respiratory failure

Pulmonary nodule or cavity, asymptomatic

Pulmonary cavity, symptomatic or chronic fibrocavitary disease

\section{Extra-pulmonary}

Cutaneous disease

Bone/joint disease, not critical location

Bone/joint disease, critical location (vertebral) or rapidly progressive

Meningitis, brain involvement

Other extrapulmonary areas, localized and not lifethreatening

Multiple areas with rapidly progressive, life-threatening disease

Positive test without clear disease

Elevated coccidioidal antibody titers without a defined focus of disease
Azole (e.g., fluconazole $400 \mathrm{mg}$ po daily) ${ }^{\mathrm{c}} \quad 3-6$ months

Liposomal amphotericin followed by azole ${ }^{c}>1$ year

None

NA

Azole, consider surgical options ${ }^{c}$

Variable

Azole (e.g., fluconazole $400-800 \mathrm{mg}$ po $\quad>1$ year

daily) ${ }^{c}>1$ year

Azole (e.g., fluconazole 400-800 mg po $>1$ year

daily) ${ }^{c}>1$ year

Liposomal amphotericin followed by azole ${ }^{c}>1$ year

High dose fluconazole $(800-1200 \mathrm{mg} / \text { day })^{c} \quad$ Lifelong

Azole (e.g., fluconazole $800 \mathrm{mg} /$ day) ${ }^{c} \quad>1$ year

Liposomal amphotericin followed by azole ${ }^{c}>1$ year

None versus azole (fluconazole

Variabled $400 \mathrm{mg} /$ day $)^{\mathrm{d}}$
Duration of

therapy $^{a}$

NA not applicable

${ }^{a}$ Dependent on host factors (e.g., immunosuppression) and clinical response (symptoms, examination findings, radiographic findings and CF titer trends). Cases of disseminated disease should be treated with a minimal of 1 year, but most require 3 or more years of therapy, and those with brain involvement should be treated with lifelong therapy

${ }^{b}$ Debilitating disease has been classified by IDSA guidelines [80] to include weight loss $>10 \%$ of body weight, intense night sweats $>3$ weeks, infiltrates of more than half of one lung or involving both lungs, prominent or persistent hilar or peritracheal adenopathy, CF titer $>1: 16$, symptoms lasting $>2$ months, need for hospitalization, or inability to work. Additionally, patients with underlying host factor $(s)$ that increases the risk for severe or disseminated disease, treatment is warranted in the setting of a symptomatic infection. Such host conditions include cellular immunodeficiencies (transplant recipients, receipt of immunosuppressive medications, HIV with CD4 cell count of $<250 \mathrm{cells} / \mathrm{mm}^{3}$ ), diabetes, a frail health status due to age or comorbidities, or non-Caucasian races, especially African Americans and Filipinos ${ }^{c}$ Prior to azole use, evaluate for drug contraindications (e.g., pregnancy, long QT interval, etc.) as well as drug interactions ${ }^{\mathrm{d}}$ Treatment is recommended for those who are immunosuppressed (e.g., transplant recipients and HIV-positive persons with low CD4 counts of $<250$ cells $/ \mathrm{mm}^{3}$ ) 
most cases is based on cost considerations, fewer drug interactions, and excellent absorption and bioavailability. Discontinuation of antifungal therapy should be based on symptom resolution, host factors, risk for disease recurrence, and CF titers. Among solid organ transplant or allogenic hematopoietic stem cell transplant recipients, ongoing suppressive azole treatment after the acute treatment course is recommended given the high risk for relapse [80]. Similarly, ongoing therapy is advised among HIV-infected persons with low CD4 cell counts $\left(<250\right.$ cells $\left./ \mathrm{mm}^{3}\right)$. Antifungal therapy can typically be discontinued in HIV-infected persons after 3-6 months if there is clinical response, a CD4 cell count $\geq 250$ cells $/ \mathrm{mm}^{3}$, and a suppressed HIV RNA level [66].

Overall, antifungal treatment for uncomplicated coccidioidal pneumonia should be individualized. All patients, regardless of medication use, should receive patient education (regarding the natural history of the disease and symptomatology that may signify complicated or disseminated course), clinical followup, and supportive measures (e.g., reconditioning physical therapy) [80].

Regarding treatment of other pulmonary forms of the disease, diffuse pneumonia with respiratory failure is typically treated with amphotericin until stabilization, followed by de-escalation to an azole for at least 1 year (Table 1). Presence of an asymptomatic pulmonary nodule does not require antifungal therapy; of note, differentiation between a postcoccidioidal nodule versus another cause (e.g., malignancy) may require biopsy. A pulmonary cavity can occur and, in asymptomatic cases, antifungal therapy is not warranted [80]. However, if a cavity persists for $>1$ year, surgical resection can be considered, especially if there is concern for future complications, such as rupture or development of a pneumothorax based on the cavity location. Those with symptomatic cavitary disease (due to ongoing cough or hemoptysis) are treated with oral antifungal therapy, but surgical management should be considered, especially in cases of ongoing symptoms despite antifungal therapy or if symptoms recur whenever antifungal treatment is stopped. Among patients who are unable or unwilling to undergo surgery, chronic antifungal therapy may be needed for symptom control. Finally, chronic fibrocavitary disease is managed with oral azole antifungal agents and possible surgical approaches among those with ongoing, non-resolving symptoms.

\section{Extrapulmonary Disease}

All cases of extrapulmonary coccidioidomycosis (e.g., skin, bone, joint, and other locations) should be treated, as the disease is typically progressive and seldom resolves on its own [80] (Table 1). There are no comparative trials of amphotericin versus azoles for disseminated disease, but, given the toxicities associated with the former agent, therapy is typically accomplished using an oral azole. For patients with rapidly progressive disease, life-threatening disease, involvement of critical locations (e.g., vertebral infection), or azole failure, treatment is with amphotericin, and then later de-escalated to an azole once clinical stability or improvement is achieved. Therapy for extrapulmonary disease is for a minimum duration of 1 year but is often longer ( 3 or more years) and should be informed by clinical findings, CF titers, and the immunocompetence of the host.

Coccidioidal meningitis (CM) is the most severe form of coccidioidomycosis and is typically treated with oral fluconazole. Of note, intravenous amphotericin has poor CSF penetration and is not an effective treatment for CM. Dosing of fluconazole typically ranges from 400 to $1200 \mathrm{mg}$ daily, typically at a starting dose of $800 \mathrm{mg}$ daily. Patients who do not respond to azole therapy may benefit from intrathecal amphotericin B; dosing and administration should be done in coordination with an expert in the field [113]. Posaconazole and voriconazole have been used in refractory cases [123, 124, 129]. Hydrocephalus can complicate CNS disease and typically requires neurosurgical consultation and placement of an internal ventricular shunt. Patients diagnosed with $\mathrm{CM}$ should be given life-long antifungal treatment, given the high risk for relapse and life-altering complications including stroke and death [80]. 


\section{Treatment during Pregnancy}

Pregnant women represent a special population in terms of the management of all forms of coccidioidomycosis. First, they are at high risk for severe, complicated disease. Secondly, treatment is complicated by the potential for teratogenicity of azoles during early pregnancy. Per IDSA guidelines, options include amphotericin, no therapy with close monitoring, or use of an azole after the first trimester. For those with $\mathrm{CM}$, intrathecal amphotericin B may be an option. Further guidance on the management of coccidioidomycosis during pregnancy is available in the IDSA guidelines [80].

\section{Elevated Coccidioidal Antibody Titers Without A Defined Focus of Disease}

Occasionally, detection of a positive coccidioidal antibody is found without symptoms or signs of a defined fungal infection. In these settings, a review of host risk factors for severe disease, a thorough physical examination, and CAR should be performed. Any foci of possible infection should be investigated. Among those with isolated positive coccidioidal antibody testing who are immunosuppressed (e.g., transplant recipients and HIV-positive persons with low CD4 counts of $<250$ cells $/ \mathrm{mm}^{3}$ ) treatment with fluconazole $400 \mathrm{mg}$ daily is recommended, since there is a notable risk for impending development of clinical illness and progression (Table 1) [66, 153]. Among the immunocompetent host, close follow-up is advised.

\section{Additional Management Strategies}

Among patients with immunosuppression due to medication(s), a reduction in the dosage or discontinuation of the immunosuppressant(s) should be considered if it would not lead to graft-versus-host disease, organ rejection, or significant relapse/flare of their underlying medical condition [80]. Persons who are immunosuppressed due to HIV infection should have treatment with antiretroviral medications to improve their cellular immunity.

Surgery should be considered for complicated bone and/or joint disease, including for debridement and stabilization purposes.
Surgical consultation is also important in cases of spinal instability, spinal cord/nerve root compression, and/or paraspinal or epidural abscesses.

Interferon gamma administration has been utilized as adjunct to antifungal agents in case reports of refractory disease [70, 123, 154]. A dose of $50 \mu \mathrm{g} / \mathrm{m}^{2}$ subcutaneously 3 times weekly has been associated with improvement; however, robust data are lacking [21].

\section{CLINICAL FOLLOW-UP}

Patient follow-up is important to ensure resolution of clinical signs/symptoms and to monitor for potential development of relapsing or disseminating disease. For those on treatment, monitoring for drug-related adverse events, drug interactions, and TDM when applicable are important. The time frame of follow-up visits should be individualized, based on disease severity, host factors, and treatment plans. In general, cases should be followed for a notable duration of time (e.g., 2 years among untreated persons and 2 years after treatment ends among those treated), given the risk for relapse and/or dissemination [21]. Follow-up should include clinical assessments as well as CF titers to ensure declining values over time. In general, CF titers are performed every 4-8 weeks, with lengthening of the time frame as the patient improves; of note, changes in titers often take weeks [155]. An increase of two doubling dilution steps in the titer is of clinical significance; for example, an increase from 1:8 to $1: 32$ would suggest worsening of the disease, and should be investigated in terms of new signs or symptoms, medication compliance, adequate absorption, TDM for specific azoles, and further diagnostic imaging studies or specimen collection (e.g., biopsy of a new lesion) as dictated by clinical findings. For those with pulmonary involvement, a follow-up CAR is warranted to re-establish a new baseline and document any residual changes that may be helpful for future medical decision-making.

Patients with a history of coccidioidomycosis who subsequently become immunosuppressed or women who become pregnant should have 
re-assessment of their fungal disease status. This should include a history and physical examination, CF titer, and consideration for imaging studies based on prior disease involvement and current symptomatology.

The duration of antifungal therapy is not standardized, but most experts and guidelines recommend 3-6 months for typical pulmonary disease and at least 1 year for diffuse pneumonia or any form of extrapulmonary disease, but the latter conditions often require longer treatment courses of 3 or more years [21, 80]. For CM, therapy is lifelong [80]. The total length of therapy should be individualized, and depends on symptom resolution, follow-up imaging and examination findings, and trends in CF titers over time with a goal CF titer of $<1: 2$ for at least 6 months prior to medication discontinuation. Clinical decision-making in challenging cases should involve an expert in the field.

\section{DISEASE IMPACT}

While many cases of coccidioidomycosis are asymptomatic or self-limited, there a significant burden of symptomatic disease annually. An interview study in Arizona of coccidioidomycosis cases found that the median time to diagnosis was 23 days, the median duration of illness was 120 days, the median number of days lost from work was 14 days, and the median time until activities of daily living were resumed was 47 days [156]. Another study among patients with primary pulmonary disease found that the median time from symptom onset to $50 \%$ reduction and to complete resolution were 9 weeks and 18 weeks, respectively, regardless of antifungal therapy [150]. Even among otherwise healthy adults, convalescence is often lengthy, and lingering fatigue may continue for months.

Another study in Arizona estimated that the total lifetime costs for the 10,359 cases of coccidioidomycosis diagnosed in 2019 was US\$736 million. Direct costs were $\$ 671$ million and accounted for over $90 \%$ of expenditures, with $\$ 65$ million in indirect costs. Disseminated infection produces the highest economic burden and cases of primary uncomplicated pneumonia had the lowest burden, with the latter costing an estimated $\$ 23,200$ in direct costs and $\$ 1300$ in lost wages. The average lifetime direct costs across all Valley Fever manifestations were $\$ 64,800$, with an additional $\$ 6300$ for indirect costs for each person diagnosed in Arizona during 2019 [157].

\section{NOVEL CO-INFECTIONS}

With the emergence of the novel coronavirus, SARS-CoV-2, a number of co-infections with Coccidioides spp. have been described. Both pathogens are occurring at increasing rates and share many of the same predisposing factors for severe disease, including older age, poorly controlled diabetes, immunosuppression, racial minority status, and pregnancy [158].

Patients with coccidioidomycosis infection may have pulmonary sequalae that place them at higher risk for severe COVID. Similarly, those with severe COVID are at risk for developing fungal infections, especially with receipt of immunosuppressive agents (e.g., dexamethasone, tocilubmab) as COVID therapies. Additionally, SARS-CoV-2 may cause host immune dysregulation with high levels of inflammatory cytokines, lymphopenia, and T-cell depletion, all of which may predispose to fungal infections.

While the exact role of COVID in potentially increasing Coccidioides infections or reactivation remains incompletely defined, cases of co-infection have been reported in the literature. A review of systemic mycoses in the setting of SARS-CoV-2 infection reported two coccidioidal cases in California, one treated with fluconazole and the other without; there were no reported fatalities [159]. An additional case of accelerated progression of disseminated coccidioidomycosis following SARS-CoV-2 has been published [160]. The full impact of SARS-CoV-2 on coccidioidomycosis cases and disease severity awaits further studies. 


\section{PREVENTION}

Preventive strategies for coccidioidomycosis include avoiding outdoor activities when dust is present, and, if outside exposure is unavoidable, wearing a respirator. Wetting the soil before soil-disruptive activities has also been advised. Education of residents and travelers to endemic areas (particularly those who are at increased risk for severe or disseminated disease) regarding preventive strategies as well as disease symptomatology is recommended.

Prophylactic use of antifungals among highrisk, immunosuppressed persons who reside in highly endemic areas is a rapidly evolving area of interest. Transplant recipients residing in endemic areas without evidence of active coccidioidomycosis are recommended to receive prophylaxis with fluconazole $200 \mathrm{mg}$ daily for at least 6-12 months post-transplant [80]. Whether prophylaxis should be extended beyond this time frame requires further study, but a study among lung transplant recipients living in an endemic area $(n=493)$ showed low rates of active disease in the setting of ongoing, life-long prophylaxis [161].

Pre-emptive testing for Coccidioides spp. is recommended among specific groups residing in endemic locations or with history of past exposure. For example, testing is advised prior to stem cell or organ transplantation. For those who will be receiving biological response modifiers without a history of infection, guidelines recommend screening prior to immunosuppressant initiation followed by clinical followup for any new signs or symptoms; ongoing serologic screening or antifungal prophylaxis is not recommended if they remain asymptomatic and are seronegative.

HIV-infected persons in endemic areas are recommended to undergo annual testing using serology and chest radiography [80]. For asymptomatic HIV patients who are serologically negative, primary antifungal therapy is not recommended. Rather, annual serologic testing using an EIA or immunodiffusion are recommended. Those with HIV infection and a CD4 count $<250$ cells $/ \mathrm{mm}^{3}$ with a new positive IgM or IgG serology despite the absence of symptoms are recommended to receive ongoing therapy (fluconazole $400 \mathrm{mg}$ po daily) until the CD4 is $>250$ cells $/ \mathrm{mm}^{3}$ for at least 6 months. For symptomatic patients, treatment is provided as outlined above [66].

For persons at high risk for complicated disease who may be considering residence and among military personnel or prisoners who may be stationed or incarcerated in endemic areas, the use of serologic testing and/or skin testing may be useful to evaluate for evidence of preexisting immunity and risk for incident infection.

Finally, regarding accidental laboratory exposure to Coccidioides spp., antifungal administration is recommended per the existing literature [162].

Regarding a vaccine to protect against $\mathrm{Coc}$ cidioides spp. infection, despite earnest efforts, no vaccine currently exists [163]. An effective vaccine is believed possible, since patients who have recovered from initial infection are thought to be protected for life. The goal of a successful vaccine would include prevention of both symptomatic and disseminated forms of the disease, especially among immunosuppressed persons and other persons at high risk. Hence, the niche for the vaccine would be among high-risk individuals, including outdoor workers, military personnel, non-Caucasians, and immunosuppressed hosts living in or traveling to endemic areas.

Based on the current understanding of effective immune responses against Coccidioides spp., successful protection would require T-cellmedicated immune responses (TH1 and TH17). The first experimental anti-Coccidioides vaccine developed was a formalin-killed spherule (FKS) vaccine that demonstrated promising results in mice in the 1960s [164, 165]. However, human trials conducted in the 1990s found no differences between the FKS-vaccinated and placebo groups [166], likely due to the dose reduction made to the formulation to address severe injection site reactions.

The approach for a coccidioidal vaccine subsequently focused on potential antigens that could be recombinantly produced and included in a vaccine. Conserved proteins present in both species have been identified, but which ones 
elicit protective immune responses remains unclear. Several recombinant vaccine candidates have been developed [13], including one using a construct of three antigens (Ag2-PRA, Cs-Ag, and Pmp-1) along with an adjuvant, but all are in early phases of development. Another candidate is a live mutant strain of $C$. posadasii that has the virulence factor encoded by the CPS1 gene deleted [167]. A major constraint to the development of both novel treatments and vaccines against coccidioidomycosis is the lack of commercial incentive and public interest. Strong governmental, philanthropical, and pharmaceutical partnerships will likely be needed to ensure progress towards an effective vaccine, a goal that is likely decades in the future.

\section{CONCLUSIONS}

Over the past century, much has been learned about coccidioidomycosis, which is endemic to the valleys of California ("Valley Fever") and other desert locations in the Western Hemisphere. The geographic distribution of the causative pathogens, C. immitis and C. posadasii, appear to be expanding and the incidence of the disease is rising. Although most coccidioidal infections are asymptomatic, coccidioidomycosis is a common cause of CAP and may disseminate through the body (especially to the skin, bone/joints, and CNS), causing notable health and economic repercussions. While the clinical features and diagnosis of the disease are well described, much is to be gained regarding more potent and safer antifungal agents against this orphan disease. As antifungals are developed, studies on their potential activity and role in coccidioidomycosis are advocated. A vaccine for preventing coccidioidomycosis among exposed persons, especially those at high risk of disseminated disease, is needed, but will likely not be realized until far in the future. An increased public awareness and interest in this disease is needed, as well as a renewed sense of urgency for novel treatments and preventive strategies for this important regional infection of global implications.

\section{ACKNOWLEDGEMENTS}

Funding. No funding or sponsorship was received for this paper or the publication of this article.

Authorship. The named author met the International Committee of Medical Journal Editors (ICMJE) criteria for authorship for this article, takes responsibility for the integrity of the work as a whole, and has given her approval for this version to be published.

Author Contributions. The entire paper was written by the sole author.

Disclosures. Dr. Nancy Crum declares no conflict of interest.

Compliance with Ethics Guidelines. This article is based on previously conducted studies and does not contain any new studies with human participants or animals performed by any of the author.

Open Access. This article is licensed under a Creative Commons Attribution-NonCommercial 4.0 International License, which permits any non-commercial use, sharing, adaptation, distribution and reproduction in any medium or format, as long as you give appropriate credit to the original author(s) and the source, provide a link to the Creative Commons licence, and indicate if changes were made. The images or other third party material in this article are included in the article's Creative Commons licence, unless indicated otherwise in a credit line to the material. If material is not included in the article's Creative Commons licence and your intended use is not permitted by statutory regulation or exceeds the permitted use, you will need to obtain permission directly from the copyright holder. To view a copy of this licence, visit http://creativecommons.org/licenses/bync/4.0/. 


\section{REFERENCES}

1. Posadas A. Un nuevo caso de micosis fungoidea con posrospemias. An Cir Med Argent. 1892;15:585-97.

2. Hirschmann JV. The early history of coccidioidomycosis: 1892-1945. Clin Infect Dis. 2007;44: 1202-7.

3. Rixford E, Gilchrist TC. Two cases of protozoan (coccidioidal) infection of the skin and other organs. Johns Hopkins Hosp Rep. 1896;10:209-68.

4. Ophuls W. Further observations on a pathogenic mould formerly described as a protozoon (Coccidioides immitis, Coccidioides pyogenes). J Exp Med. 1905;6:443-85.

5. Smith CE, Beard RR. Varieties of coccidioidal infection in relation to the epidemiology and control of the diseases. Am J Public Health Nations Health. 1946;36:1394-402.

6. Hirschmann JV. Charles Edward Smith: Coccidioidomycologist and public health leader. J Med Biogr. 2020;28:24-30.

7. Fisher MC, Koenig GL, White TJ, Taylor JW. Molecular and phenotypic description of Coccidioides posadasii sp. nov., previously recognized as the non-California population of Coccidioides immitis. Mycologia. 2002;94:73-84.

8. Sharpton TJ, Rounsley SD, Gardner MJ, et al. Comparative genomic analyses of the human fungal pathogens Coccidioides and their relatives. Genome Res. 2009;19:1722-31.

9. Nguyen C, Barker BM, Hoover S, et al. Recent advances in our understanding of the environmental, epidemiological, immunological, and clinical dimensions of coccidioidomycosis. Clin Microbiol Rev. 2013;26:505-25.

10. Hamm PS, Hutchison MI, Leonard P, Melman S, Natvig DO. First analysis of human Coccidioides isolates from New Mexico and the southwest Four Corners region: Implications for the distributions of C. posadasii and C. immitis and human groups at risk. J Fungi (Basel). 2019;5:74.

11. Neafsey DE, Barker BM, Sharpton TJ, et al. Population genomic sequencing of Coccidioides fungi reveals recent hybridization and transposon control. Genome Res. 2010;20:938-46.

12. Chow NA, Kangiser D, Gade L, et al. Factors influencing distribution of Coccidioides immitis in soil, Washington State 2016. mSphere. 2021;6: e0059821.
13. Kirkland TN, Fierer J. Coccidioides immitis and posadasii; a review of their biology, genomics, pathogenesis, and host immunity. Virulence. 2018;9: 1426-35.

14. Laniado-Laborin R. Expanding understanding of epidemiology of Coccidioidomycosis in the Western Hemisphere. Ann N Y Acad Sci. 2007;1111: 19-34.

15. Johnson SM, Carlson EL, Fisher FS, Pappagianis D. Demonstration of Coccidioides immitis and Coccidioides posadasii DNA in soil samples collected from Dinosaur National Monument. Utah Med Mycol. 2014;52:610-7.

16. Perera P, Stone S. Coccidioidomycosis in workers at an archeologic site - Dinosaur National Monument, Utah, June-July 20001. Ann Emerg Med. 2002;39: 566-9.

17. Marsden-Haug N, et al. Coccidioidomycosis acquired in Washington State. Clin Infect Dis. 2013;56:847-50.

18. Hawryluk M. Traces of Valley fever fungus found in central Oregon. The Bulletin [cited 2016 Jul 28]. http://www.bendbulletin.com/home/4524652151/traces-of-valley-fever-fungus-found-in-central. Accessed January 22, 2022.

19. Benedict $\mathrm{K}$, Ireland $\mathrm{M}$, Weinberg MP, et al. Enhanced surveillance for coccidioidomycosis, 14 US States, 2016. Emerg Infect Dis. 2018;24:1444-52.

20. Morrow W. Holocene coccidioidomycosis: valley fever in early Holocene bison (Bison antiquus). Mycologia. 2006;98:669-77.

21. Johnson RH, Sharma R, Kuran R, Fong I, Heidari A. Coccidioidomycosis: a review. J Investig Med. 2021;69:316-23.

22. Crum N, Lamb C, Utz G, et al. Coccidioidomycosis outbreak among United States Navy SEALs training in a Coccidioides immitis-endemic area-Coalinga, California. J Infect Dis. 2002;186:865-8.

23. Bowers JR, Parise KL, Kelley EJ, et al. Direct detection of Coccidioides from Arizona soils using CocciENV, a highly sensitive and specific real-time PCR assay. Med Mycol. 2019;57:246-55.

24. Gorris ME, Caballero Van Dyke MC, Carey A. A Review of Coccidioides research, outstanding questions in the field, and contributions by women scientists. Clin Infect Dis. 2007;45:562-8.

25. Del Rocio-Reyes-Montes M, Perez-Huitron MA, Ocana-Monroy JL, et al. The habitat of Coccidioides spp. and the role of animals as reservoirs in 
disseminators to nature. BMC Infect Dis. 2016;16: 550-8.

26. Centers for Disease Control and Prevention (CDC). Increase in reported coccidioidomycosis-United States, 1998-2011. Morb Mortal Wkly Rep. 2013;62: 217-21.

27. Centers for Disease Control and Prevention. Valley fever (coccidiomycosis) statistics. 2020 [cited 2020 May 14]. https://www.cdc.gov/fungal/diseases/ coccidioidomycosis/ statistics.html. Accessed January $20,2022$.

28. Cooksey GS, Nguyen A, Knutson K, et al. Notes from the field: increase in coccidioidomycosisCalifornia, 2016. Morb Mortal Wkly Rep. 2017;66: 833.

29. California Department of Public Health. Epidemiological summary of coccidioidomycosis in California, 2018. https://www.cdph.ca.gov/Programs/CID/ DCDC/Pages/Coccidioidomycosis.aspxAccessed January 20, 2022.

30. Sondermeyer Cooksey GL, Nguyen A, Vugia D, Jain S. Regional analysis of coccidioidomycosis incidence-California, 2000-2018. Morb Mortal Wkly Rep. 2020;69:1817-21.

31. Caballero Van Dyke MC, Thompson GR, Galgiani JN, Barker BM. The rise of Coccidioides: forces against the dust devil unleashed. Front Immunol. 2019;10:2188.

32. Gilford MA, Bus WC, Douds RJ. Annual report: Kern County Department of Public Health for the fiscal year July 1, 1936 to June 30, 1937. Kern County Department of Public Health. 1937. 47-52.

33. Edwards PQ, Palmer CE. Prevalence of sensitivity to coccidioidin, with special reference to specific and nonspecific reactions to coccidioidin and to histoplasmin. Dis Chest. 1957;31:35-60.

34. Maddy KT. The geographic distribution of Coccidioides immitis and possible ecologic implications. Ariz Med. 1958;15:178-88.

35. Dodge RR, Lebowitz MD, Barbee RA, et al. Estimates of $C$. immitis infection by skin test reactivity in an endemic community. Am J Public Health. 1985;75: 863-5.

36. Larwood TR. Coccidioidin skin testing in Kern County, California: decrease in infection rate over 58 years. Clin Infect Dis. 2000;30:612-3.

37. Crum NF, Potter M, Pappagianis D. Seroincidence of coccidioidomycosis during military desert training exercises. J Clin Microbiol. 2004;42:4552-5.
38. Petersen LR, Marshall SL, Barton-Dickson C, et al. Coccidioidomycosis among workers at an archeological site, northeastern Utah. Emerg Infect Dis. 2004;10:637-42.

39. Freedman M, Jackson BR, McCotter O, Benedict K. Coccidioidomycosis outbreaks, United States and worldwide, 1940-2015. Emerg Infect Dis. 2018;24: 417-23.

40. Das R, McNary J, Fitzsimmons K, et al. Occupational coccidioidomycosis in California: outbreak investigation, respirator recommendations, and surveillance findings. J Occup Environ Med. 2012;54: 564-71.

41. Cummings KC, et al. Point-source outbreak of coccidioidomycosis in construction workers. Epidemiol Infect. 2010;138:507-11.

42. Wilken JA, Sondermeyer G, Shusterman D, et al. Coccidioidomycosis among workers constructing solar power farms, California, USA, 2011-2014. Emerg Infect Dis. 2015;21:1997-2005.

43. Laws RL, Jain S, Sondermeyer Cooksey G, et al. Coccidioidomycosis outbreak among inmate wildland firefighters: California, 2017. Am J Ind Med. 2021;64:266-73.

44. Crum-Cianflone NF. Coccidioidomycosis in the US Military: a review. Ann N Y Acad Sci. 2007;1111: 112-21.

45. Standaert SM, Schaffner W, Galgiani JN, et al. Coccidioidomycosis among visitors to a Coccidioides immitis-endemic area: an outbreak in a military reserve unit. J Infect Dis. 1995;171:1672-5.

46. Centers for Disease Control and Prevention (CDC). Public health dispatch: Coccidioidomycosis among persons attending the World Championship of Model Airplane Flying-Kern County, California, October 2001. Morb Mort Wkly Rep. 2001;50: 1106-7.

47. Stern NG, Galgiani JN. Coccidioidomycosis among scholarship athletes and other college students, Arizona, USA. Emerg Infect Dis. 2010;16:321-3.

48. Diaz JH. Travel-related risk factors for coccidioidomycosis. J Travel Med. 2018; 25(1).

49. Gorris ME, Neumann JE, Kinney PL, Sheahan M, Sarofim MC. Economic valuation of coccidioidomycosis (Valley Fever) projections in the United States in response to climate change. Weather Clim Soc. 2021;13:107-23.

50. Schneider E, Hajjeh RA, Spiegel RA, et al. A coccidioidomycosis outbreak following the Northridge, Calif, earthquake. JAMA. 1997;277:904-8. 
51. Flynn NM, Hoeprich PD, Kawachi MM, et al. An unusual outbreak of windborne coccidioidomycosis. N Engl J Med. 1979;301:358-61.

52. Tong DQ, Wang JXL, Gill TE, Lei H, Wang B. Intensified dust storm activity and valley fever infection in the southwestern United States. Geophys Res Lett. 2017;44:4304-12.

53. Lucas KD, Wheeler C, Mohle-Boetani JC. Coccidioidomycosis outbreaks among inmate wildland firefighters in California. In: Proceedings of the 63rd Coccidioidomycosis Study Group Annual Meeting; 2019 Apr 5-6. Sacramento, CA, USA.

54. Weaver EA, Kolivras KN. Investigating the relationship between climate and valley fever (coccidioidomycosis). EcoHealth. 2018;15:840-52.

55. Benedict K, Li Y, Molinari NAM, Jackson BR. Health care providers' testing practices for coccidioidomycosis and histoplasmosis in patients with community-acquired pneumonia-United States, 2020. Open Forum Infect Dis. 2021;8:ofab020.

56. Tartof SY, Benedict K, Xie F, et al. Testing for coccidioidomycosis among community-acquired pneumonia patients, southern California, USA. Emerg Infect Dis. 2018;24:779-81.

57. Wilson JW, Smith CE, Plunkett OA. Primary cutaneous coccidioidomycosis; the criteria for diagnosis and a report of a case. Calif Med. 1953;79:233-9.

58. Wright PW, Pappagianis D, Wilson M, et al. Donorrelated coccidioidomycosis in organ transplant recipients. Clin Infect Dis. 2003;37:1265-9.

59. Martín-Dávila P, Fortún J, López-Vélez R. Transmission of tropical and geographically restricted infections during solid-organ transplantation. Clin Microbiol Rev. 2008;21:60-96.

60. Nelson JK, Giraldeau G, Montoya JG, Deresinski S, Ho DY, Pham M. Donor-derived Coccidioides immitis endocarditis and disseminated infection in the setting of solid organ transplantation. Open Forum Infect Dis. 2016;3:ofw086.

61. Charlton V, Ramsdell K, Sehring S. Intrauterine transmission of coccidioidomycosis. Pediatr Infect Dis J. 1999;18:561-3.

62. Donovan FM, Shubitz L, Powell D, Orbach M, Frelinger J, Galgiani JN. Early events in coccidioidomycosis. Clin Microbiol Rev. 2019;33:e00112e119.

63. Ampel NM, Dols CL, Galgiani JN. Coccidioidomycosis during human immunodeficiency virus infection: results of a prospective study in a coccidioidal endemic area. Am J Med. 1993;94:235-40.
64. Ampel NM. Delayed-type hypersensitivity, in vitro T-cell responsiveness and risk of active coccidioidomycosis among HIV-infected patients living in the coccidioidal endemic area. Med Mycol. 1999;37: 245-50.

65. Masannat FY, Ampel NM. Coccidioidomycosis in patients with HIV-1 infection in the era of potent antiretroviral therapy. Clin Infect Dis. 2010;50:1-7.

66. Panel on Opportunistic Infections in Adults and Adolescents with HIV. Guidelines for the prevention and treatment of opportunistic infections in adults and adolescents with HIV: recommendations from the Centers for Disease Control and Prevention, the National Institutes of Health, and the HIV Medicine Association of the Infectious Diseases Society of America, available at: https://clinicalinfo. hiv.gov/sites/default/files/guidelines/documents/ Adult_OI.pdf. Accessed January 23, 2022.

67. Woods CW, McRill C, Plikaytis BD, et al. Coccidioidomycosis in human immunodeficiency virusinfected persons in Arizona, 1994-1997: incidence, risk factors, and prevention. J Infect Dis. 2000;181: 1428-34.

68. Crum NF, Ballon-Landa G. Coccidioidomycosis in pregnancy: case report and review of the literature. Am J Med. 2006;119:e11-17.

69. Bercovitch RS, Catanzaro A, Schwartz BS, Pappagianis D, Watts DH, Ampel NM. Coccidioidomycosis during pregnancy: a review and recommendations for management. Clin Infect Dis. 2011;53:363-8.

70. Duplessis CA, Tilley D, Bavaro M, Hale B, Holland SM. Two cases illustrating successful adjunctive interferon-gamma immunotherapy in refractory disseminated coccidioidomycosis. J Infect. 2011;63: 223-8.

71. Sampaio EP, Hsu AP, Pechacek J, et al. Signal transducer and activator of transcription 1 (STAT1) gain-of-function mutations and disseminated coccidioidomycosis and histoplasmosis. J Allergy Clin Immunol. 2013;131:1624-34.

72. Powers AE, Bender JM, Kumanovics A, et al. Coccidioides immitis meningitis in a patient with hyperimmunoglobulin $\mathrm{E}$ syndrome due to a novel mutation in signal transducer and activator of transcription. Pediatr Infect Dis J. 2009;28:664-6.

73. Vinh DC, Masannat F, Dzioba RB, Galgiani JN, Holland SM. Refractory disseminated coccidioidomycosis and mycobacteriosis in interferongamma receptor 1 deficiency. Clin Infect Dis. 2009;49:e62-5. 
74. Vinh DC, Schwartz B, Hsu AP, et al. Interleukin-12 receptor beta1 deficiency predisposing to disseminated coccidioidomycosis. Clin Infect Dis. 2011;52: e99-102.

75. Santelli AC, Blair JE, Roust LR. Coccidioidomycosis in patients with diabetes mellitus. Am J Med. 2006;119:964-9.

76. Crum NF, Lederman ER, Stafford CM, Parrish JS, Wallace MR. Coccidioidomycosis: a descriptive survey of a reemerging disease. Clinical characteristics and current controversies. Med (Baltim). 2004;83:149-75.

77. Seitz AE, Prevots DR, Holland SM. Hospitalizations associated with disseminated coccidioidomycosis, Arizona and California, USA. Emerg Infect Dis. 2012;18:1476-9.

78. McCotter O, Kennedy J, McCollum J, et al. Coccidioidomycosis among American Indians and Alaska Natives 2001-2014. Open Forum Infect Dis. 2019;6:ofz052.

79. Huang JY, Bristow B, Shafir S, Sorvillo F. Coccidioidomycosis-associated deaths, United States, 1990-2008. Emerg Infect Dis. 2012;18:1723-8.

80. Galgiani JN, Ampel NM, Blair JE, et al. 2016 Infectious Diseases Society of America (IDSA) clinical practice guideline for the treatment of Coccidioidomycosis. Clin Infect Dis. 2016;63:e112-46.

81. Valdivia L, Nix D, Wright M, Lindberg E, Fagan T, Lieberman D, et al. Coccidioidomycosis as a common cause of community-acquired pneumonia. Emerg Infect Dis. 2006;12:958-62.

82. Kim MM, Blair JE, Carey EJ, Wu Q, Smilack JD. Coccidioidal pneumonia, Phoenix, Arizona, USA, 2000-2004. Emerg Infect Dis. 2009;15:397-401.

83. Benedict K, Kobayashi M, Garg S, Chiller T, Jackson BR. Symptoms in blastomycosis, coccidioidomycosis, and histoplasmosis versus other respiratory illnesses in commercially insured adult outpatientsUnited States, 2016-2017. Clin Infect Dis. 2021;73: e4336-44.

84. Horng LM, Yaghoubian S, Ram A, et al. Endocarditis due to Coccidioides spp: the seventh case. Open Forum Infect Dis. 2015;2:ofv086.

85. Truett AA, Crum NF. Coccidioidomycosis of the prostate gland: two cases and a review of the literature. South Med J. 2004;97:419-22.

86. Phillips P, Ford B. Peritoneal coccidioidomycosis: case report and review. Clin Infect Dis. 2000;30: 971-6.
87. Cunningham ET Jr, Seiff SR, Berger TG, et al. Intraocular coccidioidomycosis diagnosed by skin biopsy. Arch Ophthalmol. 1998;116:674-7.

88. Smilack JD, Argueta R. Coccidioidal infection of the thyroid. Arch Intern Med. 1998;158:89-92.

89. Brown EM, McTaggart LR, Dunn D, et al. Epidemiology and geographic distribution of blastomycosis, histoplasmosis, and coccidioidomycosis, Ontario, Canada, 1990-2015. Emerg Infect Dis. 2018;24: 1257-66.

90. Ogiso A, Ito M, Koyama M, Yamaoka H, Hotchi M, McGinnis MR. Pulmonary coccidioidomycosis in Japan: case report and review. Clin Infect Dis. $1997 ; 25: 1260-1$.

91. Jang J, Lee HJ, Lee I, Cho YK, Kim HJ, Sohn KH. The first imported case of pulmonary coccidioidomycosis in Korea. J Korean Med Sci. 1999;14:206-9.

92. Chandesris MO, et al. Coccidioidomycosis: an imported invasive fungal disease in France. Med Mal Infect. 2008;38:336-42.

93. Asgari N, Hahné S, Heiskanen-Kosma T, Heli Siikamäki, Morgan M. . Cases of coccidioidomycosis in Europe due to exposure in California, United States. Euro Surveill. 2001;5(50):pii=2074.

94. Oberoi JK, Wattal C, Aggarwal PK, Khanna S, Basu AK, Verma K. Pulmonary coccidioidomycosis in New Delhi. India. Infection. 2012;40:699-702.

95. Stoney RJ, Esposito DH, Kozarsky P, et al. Infectious diseases acquired by international travellers visiting the USA. J Travel Med. 2018;25:10.

96. Thompson GR, Sharma S, Bays DJ. Coccidioidomycosis: Adenosine deaminase levels, serologic parameters, culture results, and polymerase chain reaction testing in pleural fluid. Chest. 2013;143: 776-81.

97. Sakata KK, Grys TE, Chang YH, Vikram HR, Blair JE. Serum procalcitonin levels in patients with primary pulmonary coccidioidomycosis. Ann Am Thorac Soc. 2014;11:1239-43.

98. Fierer J, Burton DW, Haghighi P, et al. Hypercalcemia in disseminated coccidioidomycosis: expression of parathyroid hormone-related peptide is characteristic of granulomatous inflammation. Clin Infect Dis. 2012;55:e61-6.

99. Thompson GR, Bays DJ, Johnson SM, Cohen SH, Pappagianis D, Finkelmanc MA. Serum $(1 \rightarrow 3)-\beta-d-$ glucan measurement in coccidioidomycosis. J Clin Microbiol. 2012;50:3060-2. 
100. Blair JE, Currier JT. Significance of isolated positive IgM serologic results by enzyme immunoassay for coccidioidomycosis. Mycopathologia. 2008;166: 77-82.

101. Thompson GR. Pulmonary Coccidioidomycosis. Semin Respir Crit Care Med. 2011;32:754-63.

102. Pappagianis D, Zimmer BL. Serology of coccidioidomycosis. Clin Microbiol Rev. 1990;3:247-68.

103. Donovan FM, Ramadan FA, Khan SA, et al. Comparison of a novel rapid lateral flow assay to enzyme immunoassay results for early diagnosis of Coccidioidomycosis. Clin Infect Dis. 2021;73:e2746-53.

104. Jackson NR, Blair JE, Ampel NM. Central nervous system infections due to coccidioidomycosis. J Fungi (Basel). 2019;5:54.

105. Binnicker MJ, Buckwalter SP, Eisberner JJ, et al. Detection of Coccidioides species in clinical specimens by real-time PCR. J Clin Microbiol. 2007;45: 173-8.

106. Vucicevic D, Blair JE, Binnicker MJ, et al. The utility of Coccidioides polymerase chain reaction testing in the clinical setting. Mycopathologia. 2010;170: 345-51.

107. Langelier C, Baxi DM, Iribarne D, Chin-Hong P. Beyond the superficial: Coccidioides immitis fungaemia in a man with fever, fatigue and skin nodules: a case of an emerging and evolving pathogen. BMJ Case Rep. 2014. https://doi.org/10.1136/bcr2014-205333.

108. Padhye AA, Smith G, McLaughlin D, Standard PG, Kaufman L. Comparative evaluation of a chemiluminescent DNA probe and an exoantigen tests for rapid identification of Blastomyces dermatitidis and Coccidioides immitis. J Clin Microbiol. 1994;32: 867-70.

109. Wack EE, Ampel NM, Sunenshine RH, Galgiani JN. The return of delayed-type hypersensitivity skin testing for coccidioidomycosis. Clin Infect Dis. 2015;61:787-91.

110. Thompson JR, Lewis JS, Nix DE, Patterson TF. Current concepts and future directions in the pharmacology and treatment of coccidioidomycosis. Med Mycol. 2019;57:S76-84.

111. González GM, Tijerina R, Najvar LK, Bocanegra R, Rinaldi MG, Graybill JR. Efficacies of Amphotericin B (AMB) Lipid Complex, AMB Colloidal Dispersion, Liposomal AMB, and Conventional AMB in treatment of murine coccidioidomycosis. Antimicrob Agents Chemother. 2004;48:2140-3.
112. Sidhu R, Lash DB, Heidari A, Natarajan P, Johnson RH. Evaluation of amphotericin b lipid formulations for treatment of severe coccidioidomycosis. Antimicrob Agents Chemother. 2018;62:e02293e2317.

113. Stevens DA, Shatsky SA. Intrathecal amphotericin in the management of coccidioidal meningitis. Semin Respir Infect. 2001;16:263-9.

114. Zhu ES, Thompson GR, Kreulen C, Giza E. Amphotericin B-impregnated bone cement to treat refractory coccidioidal osteomyelitis. Antimicrob Agents Chemother. 2013;57:6341-3.

115. Galgiani JN, Catanzaro A, Cloud GA, et al. Comparison of oral fluconazole and itraconazole for progressive, nonmeningeal coccidioidomycosis. A randomized, double-blind trial Mycoses Study. Group Ann Intern Med. 2000;133:676-86.

116. Bérard A, Sheehy O, Zhao J-P, Gorgui J, Bernatsky S, Soares de Moura C, Abrahamowicz M. Associations between low- and high-dose oral fluconazole and pregnancy outcomes: 3 nested case-control studies. CMAJ. 2019;191:E179-87.

117. Molgaard-Nielsen D, Pasternak B, Hviid A. Use of oral fluconazole during pregnancy and the risk of birth defects. N Engl J Med. 2013;369:830-9.

118. Hoffmann WJ, McHardy I, Thompson GR 3rd. Itraconazole induced hypertension and hypokalemia: mechanistic evaluation. Mycoses. 2018;61: 337-9.

119. Haylett AK, Felton S, Denning DW, Rhodes LE. Voriconazole-induced photosensitivity: photobiological assessment of a case series of 12 patients. $\mathrm{Br} \mathrm{J}$ Dermatol. 2013;168:179-85.

120. Pascual A, Calandra T, Bolay S, Buclin T, Bille J, Marchetti O. Voriconazole therapeutic drug monitoring in patients with invasive mycoses improves efficacy and safety outcomes. Clin Infect Dis. 2008;46:201-11.

121. Kim MM, Vikram HR, Kusne S, Seville MT, Blair JE. Treatment of refractory coccidioidomycosis with voriconazole or posaconazole. Clin Infect Dis. 2011;53:1060-6.

122. Freifeld A, Proia L, Andes D, et al. Voriconazole use for endemic fungal infections. Antimicrob Agents Chemother. 2009;53:1648-51.

123. De la Hoz A, Malek A, Hasbun R. Interferon- $\gamma$ and voriconazole combined therapy for refractory meningeal coccidioidomycosis in a patient with interferon- $\gamma$ deficiency. ID Cases. 2020;21:e00835. 
124. Cortez KJ, Walsh TJ, Bennett JE. Successful treatment of coccidioidal meningitis with voriconazole. Clin Infect Dis. 2003;36:1619-22.

125. Thompson GR, Beck KR, Patt M, Kratschmar DV, Odermatt A. Posaconazole-induced hypertension due to inhibition of $11 \beta$-Hydroxylase and $11 \beta$ Hydroxysteroid dehydrogenase 2. J Endocr Soc. 2019;3:1361-6.

126. Rüping MJ, Albermann N, Ebinger F, et al. Posaconazole concentrations in the central nervous system. J Antimicrob Chemother. 2008;62:1468-70.

127. Catanzaro A, Cloud GA, Stevens DA, Levine BE, Williams PL, Johnson RH. Safety, tolerance, and efficacy of posaconazole therapy in patients with nonmeningeal disseminated or chronic pulmonary coccidioidomycosis. Clin Infect Dis. 2007;45:562-8.

128. Anstead GM, Corcoran G, Lewis J, Berg D, Graybill JR. Refractory coccidioidomycosis treated with posaconazole. Clin Infect Dis. 2005;40:1770-6.

129. Schein R, Homans J, Larsen RA, Neely M. Posaconazole for chronic refractory coccidioidal meningitis. Clin Infect Dis. 2011;53:1252-4.

130. Naeem F, Laningham F, Kuzmic B, Clerkin P, McCarty J. Isavuconazole as salvage therapy for refractory pediatric coccidioidal meningitis. Pediatr Infect Dis J. 2021;40:e128-31.

131. Heidari A, Quinlan M, Benjamin DJ, Laurence B, Mu A, Ngai T, et al. Isavuconazole in the treatment of coccidioidal meningitis. Antimicrob Agents Chemother. 2019;63:e02232-e2318.

132. Thompson GR, Rendon A, Ribeiro Dos Santos R, et al. Isavuconazole treatment of cryptococcosis and dimorphic mycoses. Clin Infect Dis. 2016;63: 356-62.

133. Davis MR, Chang S, Gaynor P, McCreary EK, Allyn $P$. Isavuconazole for treatment of refractory coccidioidal meningitis with concomitant cerebrospinal fluid and plasma therapeutic drug monitoring. Med Mycol. 2021;59:939-42.

134. Thompson GR, Barker BM, Wiederhold NP. Largescale evaluation of in vitro amphotericin $b$, triazole, and echinocandin activity against Coccidioides species from US institutions. Antimicrob Agents Chemother. 2017;61:e02634-16.

135. Nakai T, Uno J, Ikeda F, Tawara S, Nishimura K, Miyaji M. In vitro antifungal activity of micafungin (FK463) against dimorphic fungi: comparison of yeast-like and mycelial forms. Antimicrob Agents Chemother. 2003;47:1376-81.
136. Gonzalez GM, Tijerina R, Najvar LK, Bocanegra R, Luther M, Rinaldi MG, Graybill JR. Correlation between antifungal susceptibilities of Coccidioides immitis in vitro and antifungal treatment with caspofungin in a mouse model. Antimicrob Agents Chemother. 2001;45:1854-9.

137. González GM, González G, Najvar LK, Graybill JR. Therapeutic efficacy of caspofungin alone and in combination with amphotericin B deoxycholate for coccidioidomycosis in a mouse model. J Antimicrob Chemother. 2007;60:1341-6.

138. O'Shaughnessy E, Yasinskaya Y, Dixon C, et al. FDA public workshop summary-coccidioidomycosis (valley fever): Considerations for development of antifungal drugs. Clin Infect Dis. 2021. https://doi. org/10.1093/cid/ciab904.

139. Thompson GR 3rd, Lewis P, Mudge S, Patterson TF, Burnett BP. Open-label crossover oral bioequivalence pharmacokinetics comparison for a 3-day loading dose regimen and 15-day steady-state administration of SUBA-itraconazole and conventional itraconazole capsules in healthy adults. Antimicrob Agents Chemother. 2020;64:e00400e420.

140. Hnik P, Wasan EK, Wasan KM. Safety, Tolerability, and Pharmacokinetics of a novel oral amphotericin b formulation (iCo-019) following single-dose administration to healthy human subjects: an alternative approach to parenteral amphotericin b administration. Antimicrob Agents Chemother. 2020;64:e01450-e1520.

141. Wiederhold NP. Review of the novel investigational antifungal olorofim. J Fungi. 2020;6:122.

142. Wiederhold NP, Najvar LK, Jaramillo R, et al. The orotomide Olorofim is efficacious in an experimental model of central nervous system coccidioidomycosis. Antimicrob Agents Chemother. 2018. https://doi.org/10.1128/AAC.00999-18.

143. Sass G, Larwood DJ, Martinez M, Chatterjee P, Xavier MO, Stevens DA. Nikkomycin $Z$ against disseminated coccidioidomycosis in a murine model of sustained-release dosing. Antimicrob Agents Chemother. 2021;65:e0028521.

144. Shubitz LF, Roy ME, Nix DE, Galgiani JN. Efficacy of Nikkomycin $\mathrm{Z}$ for respiratory coccidioidomycosis in naturally infected dogs. Med Mycol. 2013;51: 747-54.

145. Shubitz LF, Trinh HT, Perrill RH, et al. Modeling Nikkomycin $\mathrm{Z}$ dosing and pharmacology in murine pulmonary coccidioidomycosis preparatory to phase 2 clinical trials. J Infect Dis. 2014;209: 1949-54. 
146. Gintjee TJ, Donnelley MA, Thompson GR. Aspiring antifungals: Review of current antifungal pipeline developments. J Fungi (Basel). 2020;6(1):28.

147. Levy ER, McCarty JM, Shane AL, Weintrub PS. Treatment of pediatric refractory coccidioidomycosis with combination voriconazole and caspofungin: a retrospective case series. Clin Infect Dis. 2013;56:1573-8.

148. Li RK, Rinaldi MG. In vitro antifungal activity of Nikkomycin $\mathrm{Z}$ in combination with fluconazole or itraconazole. Antimicrob Agents Chemother. 1999;43:1401-5.

149. Ampel NM, Giblin A, Mourani JP, Galgiani JN. Factors and outcomes associated with the decision to treat primary pulmonary coccidioidomycosis. Clin Infect Dis. 2009;48:172-8.

150. Blair JE, Chang YH, Cheng MR, et al. Characteristics of patients with mild to moderate primary pulmonary coccidioidomycosis. Emerg Infect Dis. 2014;20:983-90.

151. Messina JA, Maziarz EK, Galgiani J. A randomized, double-blind, placebo-controlled clinical trial of fluconazole as early empiric treatment of coccidioidomycosis pneumonia (Valley Fever) in adults presenting with community-acquired pneumonia in endemic areas (FLEET-Valley Fever). Contemp Clin Trials Commun. 2021;24:100851.

152. Thompson GR, Lunetta JM, Johnson SM, et al. Early treatment with fluconazole may abrogate the development of IgG antibodies in coccidioidomycosis. Clin Infect Dis. 2011;53:e20-4.

153. Arguinchona HL, Ampel NM, Dols CL, Galgiani JN, Mohler MJ, Fish DG. Persistent coccidioidal seropositivity without clinical evidence of active coccidioidomycosis in patients infected with human immunodeficiency virus. Clin Infect Dis. 1995;20:1281-5.

154. Kuberski TT, Servi RJ, Rubin PJ. Successful treatment of a critically ill patient with disseminated coccidioidomycosis, using adjunctive interferon-gamma. Clin Infect Dis. 2004;38:910-2.

155. McHardy IH, Dinh B-TN, Waldman S, et al. Coccidioidomycosis complement fixation titer trends in the age of antifungals. J Clin Microbiol. 2018;56: e01318-18.

156. Tsang CA, Anderson SM, Imholte SB, et al. Enhanced surveillance of coccidioidomycosis, Arizona, USA, 2007-2008. Emerg Infect Dis. 2010;16: $1738-44$.
157. Grizzle AJ, Wilson L, Nix DE, Galgiani JN. Clinical and economic burden of valley fever in arizona: an incidence-based cost-of-illness analysis. Open Forum Infect Dis. 2020;8:ofaa623.

158. Heaney AK, Head JR, Broen $K$, et al. Coccidioidomycosis and COVID-19 co-infection, United States, 2020. Emerg Infect Dis. 2021;27:1266-73.

159. Frías-De-León MG, Pinto-Almazán F, HernándezCastro R, et al. Epidemiology of mycoses in the COVID-19 pandemic. J Fungi (Basel). 2021;7:556.

160. Krauth DS, Jamros CM, Rivard SC, Olson NH, Maves RC. Accelerated progression of disseminated coccidioidomycosis following SARS-CoV-2 infection: a case report. Mil Med. 2021;186:1254-6.

161. Truong CN, Nailor MD, Walia R, Cherrier L, Nasar A, Goodlet KJ. Universal lifelong fungal prophylaxis and risk of coccidioidomycosis in lung transplant recipients living in an endemic area. Clin Infect Dis. 2021. https://doi.org/10.1093/cid/ciab752.

162. Stevens DA, Clemons KV, Levine HB, et al. Expert opinion: what to do when there is Coccidioides exposure in a laboratory. Clin Infect Dis. 2009;49: 919-23.

163. Kirkland TN. The quest for a vaccine against coccidioidomycosis: a neglected disease of the Americas. J Fungi (Basel). 2016;2:E34.

164. Levine HB, Cobb JM, Smith CE. Immunity to coccidioidomycosis induced in mice by purified spherule, arthrospore, and mycelial vaccines. Trans N Y Acad Sci. 1960;22:436-49.

165. Kong YC, Levine HB. Experimentally induced immunity in the mycoses. Bacteriol Rev. 1967;31: $35-53$.

166. Pappagianis D. Evaluation of the protective efficacy of the killed Coccidioides immitis spherule vaccine in humans. The Valley Fever Vaccine Study Group. Am Rev Respir Dis. 1993;148:656-60.

167. Ampel NM. Coccidioidomycosis: changing concepts and knowledge gaps. J Fungi (Basel). 2020;6: 354.

\section{Publisher's Note}

Springer Nature remains neutral with regard to jurisdictional claims in published maps and institutional affiliations. 